\title{
Retrievals of the Far Infrared surface emissivity over the Greenland Plateau using the Tropospheric Airborne Fourier Transform Spectrometer (TAFTS).
}

\section{Christophe Bellisario ${ }^{1}$, Helen E. Brindley ${ }^{1}$, Jonathan E. Murray ${ }^{2}$, Alan Last ${ }^{2}$, Juliet Pickering $^{2}$, R. Chawn Harlow ${ }^{3}$, Stuart Fox ${ }^{3}$, Cathryn Fox ${ }^{2,3}$, Stuart M. Newman ${ }^{3}$, Maureen Smith ${ }^{4}$, Doug Anderson ${ }^{4}$, Xianglei Huang ${ }^{5}$ and Xiuhong Chen ${ }^{5}$}

${ }^{1}$ Space and Atmospheric Physics Group, Imperial College London and National Centre for Earth Observation, SW7 2AZ, UK.

${ }^{2}$ Space and Atmospheric Physics Group, Imperial College London, SW7 2AZ, UK.

${ }^{3}$ Met Office, Fitzroy Road, Exeter, UK.

${ }^{4}$ Facility for Airborne Atmospheric Measurements (FAAM), Cranfield, UK.

${ }^{5}$ Department of Climate and Space Sciences and Engineering, the University of Michigan at Ann Arbor, Ann Arbor, Michigan, USA.

Corresponding author: Christophe Bellisario (c.bellisario@imperial.ac.uk)

\section{Key Points:}

- Retrievals of far infrared surface emissivity are reported for the first time, exploiting aircraft observations taken over Greenland.

- The retrieved emissivity reaches values as low as 0.89 over the range $360-535 \mathrm{~cm}^{-1}$, where the associated uncertainties are smallest.

- Simulations of the surface emissivity are unable to simultaneously match retrievals in the far and mid infrared.

\begin{abstract}
The Tropospheric Airborne Fourier Transform Spectrometer (TAFTS) measured near surface upwelling and downwelling radiances within the far infrared (FIR) over Greenland during two flights in March 2015. Here we exploit observations from one of these flights to provide in-situ estimates of FIR surface emissivity, encompassing the range $80-535 \mathrm{~cm}^{-1}$. The flight campaign and instrumental set-up is described as well as the retrieval method, including the quality control performed on the observations. The combination of measurement and atmospheric profile uncertainties means that the retrieved surface emissivity has the smallest estimated error over the range $360-535 \mathrm{~cm}^{-1},(18.7-27.8 \mu \mathrm{m})$, lying between 0.89 and 1 with an associated error which is of the order \pm 0.06 . Between 80
\end{abstract}

This is the author manuscript accepted for publication and has undergone full peer review but has not been through the copyediting, typesetting, pagination and proofreading process, which may lead to differences between this version and the Version of Record. Please cite this article as doi: 10.1002/2017JD027328 
and $360 \mathrm{~cm}^{-1}$, the increasing opacity of the atmosphere, coupled with the uncertainty in the atmospheric state, means that the associated errors are larger and the emissivity values cannot be said to be distinct from 1 . These FIR surface emissivity values are, to the best of our knowledge, the first ever from aircraft-based measurements. We have compared them to a recently developed theoretical database designed to predict the infrared surface emissivity of frozen surfaces. When considering the FIR alone, we are able to match the retrievals within uncertainties. However, when we include contemporaneous retrievals from the mid infrared (MIR), no single theoretical representation is able to capture the FIR and MIR behaviour simultaneously. Our results point towards the need for model improvement and further testing, ideally including in-situ characterisation of the underlying surface conditions.

\section{Introduction}

Harries et al. (2008) suggest that the far infrared (FIR: defined here as wavenumbers less than $667 \mathrm{~cm}^{-1}$ ) emission of the Earth as seen from space can be considered to be sensitive to the atmospheric state alone. They note that the absorption of water vapour is so strong in this portion of the spectrum that "the surface is entirely obscured except in the coldest and driest conditions". The exceptions occur where the water vapour column is significantly reduced, by a very cold, dry atmosphere, a raised surface such as the Tibetan plateau, or a combination of the two such as over the Greenland ice-sheet. Under these conditions atmospheric transmission in the FIR increases, allowing radiation emitted by the surface to escape to space.

Recent studies have suggested that the surface emissivity in the FIR in polar regions may play a previously unrecognised role in accelerating high latitude climate change (Feldman et al., 2014). Climate models generally make the assumption that the surface emissivity of snow and ice in the FIR is equivalent to that of an ideal blackbody, an approximation which is not consistent with the indices of refraction or geometrical properties of real materials. More realistic estimates of FIR emissivity taking these factors into account suggest that, while not acting as a perfect blackbody emitter, snow and ice are significantly more emissive than the ocean surface across this spectral range (Chen et al., 2014). Indeed, due to the very dry conditions typical of polar locations, emission from the surface is able to penetrate further through the atmosphere than would usually be the case within the FIR, escaping to space in some of the most transparent windows (Harries et al., 2008). Hence, as sea-ice melts, exposing the less emissive ocean below, the surface can retain significant additional heat, potentially accelerating further ice melt. Feldman et al. (2014) show that this 'ice-emissivity feedback' can lead to increases in decadal average Arctic surface temperatures of up to $2 \mathrm{~K}$ along with concomitant decreases of up to $15 \%$ in Arctic sea-ice extent by the 2030s.

The snow and ice emissivities described by Chen et al. (2014), hereafter denoted C14, and used in Feldman et al. (2014), are derived from theoretical modelling. In the mid-infrared (MIR) these estimates have been compared to in-situ observations from Hori et al. (2016) and show reasonable agreement (see Figure $2 \mathrm{~d}$ from $\mathrm{C} 14$ ). However, up to now, to the best of our knowledge, there has been no observationally based evaluation of their reliability across the FIR. This study thus aims to produce both the first estimation of FIR surface emissivity from 
aircraft measurements over the Greenland plateau, and the first evaluation of the $\mathrm{C} 14$ emissivity simulations in the FIR. Additional MIR measurements are also used to assess the ability of the simulations to correctly capture both FIR and MIR emissivity behaviour simultaneously.

The FAAM (Facility for Airborne Atmospheric Measurements) aircraft measurements exploited in this study were 'Flights of Opportunity' made under the auspices of the CIRCCREX/COSMICS (Cirrus Coupled Cloud-Radiation Experiment/Cold-air Outbreak and Sub-Millimetre Ice Cloud Study) projects. Here we focus on one low level flight, B898, over the Greenland ice sheet during March 2015. In section 2 we introduce the TAFTS instrument and describe the CIRCCREX/COSMICS flight and auxiliary measurements available to aid our analysis. Section 3 outlines our emissivity retrieval method, including details of the required atmosphere simulations, while in section 4 we present the retrieved FIR emissivity and its uncertainty analysis. Section 5 discusses the results and shows comparisons with the C14 database, expanding the comparison to include MIR retrievals from the Airborne Research Interferometer Evaluation System (ARIES) (Wilson et al., 1999), also flying on the FAAM aircraft. Section 6 provides our final conclusions.

\section{Flight Campaign and Instrumentation}

\subsection{The TAFTS instrument}

The Tropospheric Airborne Fourier Transform Spectrometer (TAFTS, Canas et al., 1997) is a Martin-Puplett polarising interferometer (Martin et al., 1969) with a nominal spectral coverage from $80 \mathrm{~cm}^{-1}$ to $600 \mathrm{~cm}^{-1}(125-17 \mu \mathrm{m})$ and a nominal spectral resolution of $0.12 \mathrm{~cm}^{-1}$. The TAFTS configuration is a four-port system comprised of two input and two output ports. One input port is associated with the nadir and the other with the zenith view of the instrument. The detected interferogram at each output port is a superposition of the interference signal from the input ports modulating in anti-phase, and after Fourier transform, yields a spectrum which is the difference of the two input spectra. Each output is further divided into two spectral channels using a dichroic filter. The first, 'long-wave' (LW) channel (80-300 $\left.\mathrm{cm}^{-1}\right)$ uses a GeGa photo-conductor detector, while the second 'short-wave' (SW) channel $\left(330-600 \mathrm{~cm}^{-1}\right)$ uses a SiSb photo-conductor detector. The field of view of the instrument is $\pm 0.8^{\circ}$ with an aperture diameter of $25 \mathrm{~mm}$. A liquid helium cryostat cools the detectors.

A TAFTS observation sequence includes nadir and zenith views (measuring upwelling and downwelling radiances respectively), along with periodic views of internal calibration targets. There are two pairs of these blackbody targets associated with the nadir and zenith input ports. Each pair consists of a cold (near ambient) and hot $\left(50^{\circ} \mathrm{C}\right)$ black-body which are used to isolate the nadir/zenith scenes and calibrate the spectra. Two steerable mirrors are used to alternate between the scene views and the black-bodies. The black-body temperatures are monitored using small platinum resistance thermistors. TAFTS has been successfully operated on a variety of different aircraft and from the surface during the course of many previous measurement campaigns (e.g. Cox et al., 2007, Cox et al., 2010, Green et al., 2012, Fox et al., 2015). 


\subsection{CIRCCREX/COSMICS flight B898}

In this study we make use of TAFTS scans at a reduced spectral resolution of 0.24 $\mathrm{cm}^{-1}$ to enhance instrument signal to noise, taken during Flight B898 on $19^{\text {th }}$ March 2015. The instrument was aboard the FAAM BAe 146-301 aircraft which took off from the Keflavík International Airport in Iceland at 11:57 UTC, approaching Greenland from the South-East (see Figure 1). The 5-hour flight had two objectives: the first was to perform calibration and validation of instruments on-board the Suomi NPP satellite (requiring high altitude aircraft flight runs) and the second was to characterise surface emissivity (requiring low altitude runs), both under clear conditions.

For the analysis reported here, two instruments in addition to TAFTS provided key radiometric information: a Heimann radiometer, providing narrow band radiances integrated over the range $8-14 \mu \mathrm{m}\left(714.3-1250 \mathrm{~cm}^{-1}\right)$ and ARIES. The ARIES instrument is a thermal infra-red Fourier transform spectrometer capable of viewing in the nadir or zenith, covering the spectral range $550-3000 \mathrm{~cm}^{-1}(3.3-18 \mu \mathrm{m})$ with a spectral resolution of $1 \mathrm{~cm}^{-1}$ and a field of view of $2.5^{\circ}$. ARIES has been used in numerous studies, for example, to characterise the atmospheric state (e.g. Allen et al., 2014), to assess radiative impacts (e.g. Highwood et al., 2003) and for satellite spectrometer calibration/validation (e.g. Newman et al., 2012). Here we exploit its ability to simultaneously retrieve surface temperature and MIR emissivity (Newman et al., 2005, Thelen et al., 2009).

During the out-bound high-altitude transit the Advanced Vertical Atmospheric Profiling System (AVAPS) periodically released dropsondes providing temperature and water vapour profiles; in all 11 dropsondes were released (Figure 1). In addition, at the aircraft level, the true air temperature and water vapour mixing ratio were measured with a Rosemount de-iced temperature sensor and WVSS-II hygrometer fed from a modified Rosemount inlet (Vance et al., 2015), respectively.

Figure 1(a) indicates that the measurements analysed here were obtained over the highest region of the Greenland plateau where surface elevation typically exceeds $3 \mathrm{~km}$. Near contemporaneous satellite observations show that, excepting small area to the south of the operating area, the pattern of window brightness temperature roughly maps to the topography, suggesting predominantly cloud-free conditions (Figure 1(b)), an inference reinforced by lidar observations from the aircraft. The nadir and zenith radiance measurements used in this study are those taken while at low altitude (at about $330 \mathrm{~m}$ above the ice sheet surface, corresponding to a mean height of $3595 \mathrm{~m}$ above sea level), from 14:51 to 14:52 UTC (Figure 1(b) inset). During this period, the aircraft altitude above ground level varied by less than $4 \mathrm{~m}$, minimising altitude induced variability in the water vapour column present below and above the plane. Additional radiance measurements were obtained before and after the selected observations. However, significant variations in aircraft altitude and in the water vapour volume mixing ratio observed at the aircraft level during these scans mean they were not considered optimal for the retrieval of surface emissivity. In total, over the selected period, 6 quality assured scans were integrated for each view, amounting to integration times of no more than 30 s and a scene separation of 30 s. 
Observations from ARIES were used to derive estimates of surface temperature using measurements from the 8-12 $\mu \mathrm{m}$ atmospheric window. Since TAFTS and ARIES did not take their nadir views at precisely the same time (96 s offset) the continuous measurements made by the Heimann radiometer are used as a transfer standard to translate the ARIES surface temperature retrieval to the time of the TAFTS nadir measurement. This approach yields an estimate of the surface temperature of $230.5 \pm 0.4 \mathrm{~K}$ during the TAFTS observations analysed here. MIR emissivities from ARIES are estimated simultaneously with the surface temperature as part of the retrieval approach (Newman et al., 2005, Thelen et al., 2009) and these are exploited later in this study to provide a combined MIR/FIR evaluation of the C14 emissivity simulations.

Situated at approximately $40 \mathrm{~km}$ from the low-level aircraft runs $\left(37^{\circ} \mathrm{E}, 72.6^{\circ} \mathrm{N}\right.$, white square in Figure 1), Summit station routinely launches radiosondes, providing profiles of temperature and water vapour as part of the ICECAPS observational programme (Shupe et al., 2013). Figure 2 shows the temperature (a) and water vapour (b) profiles from a Summit radiosonde launch at 14:52 UTC on March $19^{\text {th }}$, within $84 \mathrm{~s}$ of the TAFTS scans. The temperature profile displays a strong near-surface inversion and then almost isothermal behaviour. The amount of water vapour in the atmospheric layer below the aircraft is exceptionally low, at approximately $0.08 \mathrm{~mm}$, and a reasonable agreement is seen between the radiosonde and FAAM measurements at the aircraft level despite the distance between the plane and Summit station. Note that the Summit sonde is used in preference to the aircraft dropsondes due to its closer proximity in space and time to the TAFTS scans used in the emissivity retrieval.

\section{The Emissivity Retrieval Method}

\subsection{Description of the method}

Li et al. (2003) provide a comprehensive review of the numerous approaches that have been employed to derive land surface emissivity from space. Aside from identifying cloud-free scenes, there are two main barriers to an accurate retrieval. The first relates to the separation of surface temperature and emissivity contributions to the emitted surface radiation, the second, the requirement to perform an atmospheric correction in order to account for the influence of the intervening atmosphere on the measured satellite signal. In this study the second issue is substantially mitigated by the fact that the path length between the aircraft and the ground is $300 \mathrm{~m}$ or less and the water vapour content of this layer is exceptionally low. To circumvent the first issue we choose to make use of the simultaneous, but independent estimate of surface temperature provided by the scaled ARIES retrieval. We then derive the surface emissivity based on a direct inversion of the measured TAFTS radiances. The simplest approach assumes specular reflection such that the upwelling nadir radiance $L_{\mathcal{v} \text {,surf }}^{\uparrow}$ from the surface is given by:

$$
L_{v, \text { surf }}^{\uparrow}=\varepsilon_{v} B_{v}\left(T_{s}\right)+\left(1-\varepsilon_{v}\right) L_{v, \text { surf }}^{\downarrow}
$$


where $\varepsilon_{v}$ is the surface emissivity, $L_{v \text {,surf }}^{\downarrow}$ is the downwelling zenith radiance incident on the surface, $B_{v}\left(T_{s}\right)$ is the Planck emission from a surface at temperature $T_{s}$ and the $v$ subscript indicates a dependency on wavenumber (see for example Newman et al., 2005).

The upwelling radiance reaching the TAFTS instrument $L_{v, T A F T S}^{\uparrow}$ can be expressed as:

$$
L_{v, T A F T S}^{\uparrow}=\tau_{v} L_{v, \text { surf }}^{\uparrow}+E_{v}^{\uparrow}
$$

where $E_{v}^{\uparrow}$ is the upwelling atmospheric emission from the layer between the surface and the aircraft and $\tau_{v}$ the layer transmission. Finally, the downwelling zenith radiance at the surface is separated into two terms (equation 3). The first is the downwelling zenith radiance incident at the top of the layer, $L_{v, T A F T S}^{\downarrow}$, (measured directly by TAFTS) which is transmitted through the layer, the second, the downwelling atmospheric emission from the layer between the surface and the aircraft, $E_{\nu}^{\downarrow}$ :

$$
L_{v, \text { surf }}^{\downarrow}=\tau_{v} L_{v, T A F T S}^{\downarrow}+E_{v}^{\downarrow}
$$

By combining equations 1 to 3 , we can write the surface emissivity as:

$$
\varepsilon_{v}=\frac{L_{v, T A F T S}^{\uparrow}-\tau_{v}^{2} L_{v, T A F T S}^{\downarrow}-\tau_{v} E_{\nu}^{\downarrow}-E_{v}^{\uparrow}}{\tau_{v}\left[B_{v}\left(T_{S}\right)-\tau_{v} L_{v, T A F T S}^{\downarrow}-E_{\nu}^{\downarrow}\right]}
$$

Equation 4 assumes that the ground is reflecting as a specular surface. Using measurements from a wintertime measurement campaign, Harlow (2009) showed that snow surfaces display a Lambertian scattering behaviour in the microwave regime. Similarly, Guedj et al. (2010) adopted a Lambertian surface scattering assumption to improve assimilation of the Advanced Microwave Sounding Unit (AMSU) measurements during the winter season. The assumption of Lambertian reflectance has also been made in the infrared (Thome et al., 1999, Thelen et al., 2009) for unspecified surfaces. Assuming that the surface is a Lambertian reflector, the angular-integrated downwelling radiance can be approximated by a single effective incidence angle. Different angles have been suggested based on the application and wavelength range considered but they typically range from $55^{\circ}-60^{\circ}$ (Mätzler, 2005, Knuteson et al., 2004). Using this approach, the downwelling radiance can be written:

$$
L_{v, \text { surf }}^{\downarrow}=\tau_{v, \text { eff }} L_{v, T A F T S, \text { eff }}^{\downarrow}+E_{v, \text { eff }}^{\downarrow}
$$

with $\tau_{v, \text { eff }}$ and $E_{v, \text { eff }}^{\downarrow}$ being respectively the transmission and the downwelling atmospheric emission at the appropriate effective incident angle. For consistency, the downwelling radiance measured by the TAFTS instrument must also be corrected to be representative of the same angle. The emissivity in the Lambertian case thus becomes:

$$
\varepsilon_{v}=\frac{L_{v, T A F T S}^{\uparrow}-\tau_{v} \tau_{v, \text { eff }} L_{\nu, T A F T S, e f f}^{\downarrow}-\tau_{v} E_{v, \text { eff }}^{\downarrow}-E_{v}^{\uparrow}}{\tau_{\nu}\left[B_{v}\left(T_{S}\right)-\tau_{v, \text { eff }} L_{\nu, T A F T S, e f f}^{\downarrow}-E_{\nu, e f f}^{\downarrow}\right]}
$$

Therefore, to compute the FIR emissivity, in addition to the TAFTS measurements and an estimate of surface temperature, the transmission $\tau_{v}$ (or $\tau_{v, \text { eff }}$ for the Lambertian case), the upwelling atmospheric emission $E_{v}^{\uparrow}$ and the downwelling atmospheric emission $E_{\mathcal{v}}^{\downarrow}$ (or $E_{v, e f f}^{\downarrow}$ for the Lambertian case) must be simulated. Given the previous results reported in 
the microwave for the winter season, in the results presented here we focus purely on the Lambertian case. However, it is worth noting that replacing this assumption with that of specular surface reflection has very little impact on the final emissivity values that we retrieve.

The uncertainty in emissivity derived from (6) can be considered to be composed of two parts:

- A 'direct' component, $\Delta \varepsilon_{v, \text { dir }}$, consisting of the impact of uncertainties in the upwelling and downwelling TAFTS radiance measurements and that of the surface temperature observed by ARIES/Heimann on the retrieved emissivity:

$=\Delta \varepsilon_{v, \text { dir }}=\left|\frac{\partial \varepsilon_{v}}{\partial L_{v, T}^{\uparrow}}\right| \Delta L_{v, T A F T S}^{\uparrow}+\left|\frac{\partial \varepsilon_{v}}{\partial L_{v, T, e f f}^{\downarrow}}\right| \Delta L_{v, T A F T S}^{\downarrow}+\left|\frac{\partial \varepsilon_{v}}{\partial T_{S}}\right| \Delta T_{S}$

- An 'indirect' component, $\Delta \varepsilon_{v, \text { ind }}$, which expresses the impact of uncertainties in the simulated atmospheric emission/transmittance, as derived from the sonde profile data, on the retrieved emissivity.

Hence, assuming direct and indirect errors to be independent, the total uncertainty in the retrieved emissivity can be written as:

$$
\Delta \varepsilon_{v}=\sqrt{\Delta \varepsilon_{v, \text { dir }}^{2}+\Delta \varepsilon_{v, \text { ind }}^{2}}
$$

\subsection{Atmospheric simulation}

The Line-By-Line Radiative Transfer Model (LBLRTM) developed by Clough et al. (2005) is used to simulate the atmospheric emission and transmission. The version used in this study is LBLRTM v12.7, with an updated line parameter database AER version 3.5 (following HITRAN 2012, Rothman et al., (2013)).

The temperature and water vapour profiles used in the simulation are taken from the Summit radiosonde launch shown in Figure 2, scaled to the FAAM WVSS-II Rosemount measurements at the aircraft level. The radiosonde provided measurements at 42 levels between the ground and the aircraft and these are used to define the simulation levels. Concentrations of $\mathrm{CO}_{2}, \mathrm{CH}_{4}, \mathrm{NO}_{2}$ and other minor gases are scaled to recent values as reported by NOAA's Global Monitoring Division, Earth System Research Laboratory, while the ozone profile is taken from the nearest available ECMWF Interim Reanalysis (ERA-I) (Dee et al., 2011) profile in space and time. We apply a Kaiser apodisation function of resolution $0.24 \mathrm{~cm}^{-1}$, matching the TAFTS spectra, to the native $0.01 \mathrm{~cm}^{-1}$ LBLRTM output before undertaking emissivity retrievals.

Following the assumption of Lambertian surface reflectance in equation 6, an atmospheric correction for the effective angle must be applied to the measured $L_{\mathcal{v}, T A F T S}^{\downarrow}$ to obtain $L_{v, T A F T S, e f f}^{\downarrow}$. A sensitivity study using the atmospheric conditions outlined above was carried out to determine the best angle to use in order to approximate the hemispherically integrated downwelling radiance. The downwelling radiance was simulated over the full 
range of zenith angles. Then the hemispheric integrated downwelling radiance was computed according to

$$
L^{\downarrow}(v)=\int_{0}^{2 \pi} \int_{0}^{\pi / 2} L^{\downarrow}(\theta, \varphi, v) \cos (\theta) \sin (\theta) d \theta d \varphi
$$

Here, $\theta$ is the zenith angle and

isotropy. The individual zenith angle that gave the minimum difference with the angularly integrated radiance was found at each wavenumber. We found that while an angle of $55^{\circ}$ minimised residuals across the $\mathrm{SW}$, a smaller angle of $43.5^{\circ}$ minimised residuals across the LW. However, in practice using $55^{\circ}$ for the whole range has an impact of less than $0.02 \%$ on the retrieved emissivity in the LW. Therefore, using the simulations, we compute the corrected downwelling radiance as:

$$
L_{v, T A F T S, \text { eff }}^{\downarrow}=L_{\nu, T A F T S}^{\downarrow}\left(55^{\circ}\right)=\frac{L_{v, L B L R T M}^{\downarrow}\left(55^{\circ}\right)}{L_{v, L B L R T M}^{\downarrow}\left(0^{\circ}\right)} L_{v, T A F T S}^{\downarrow}\left(0^{\circ}\right)
$$

For clarity, Figure 3 summarises the overall FIR emissivity retrieval approach, identifying the various inputs employed. Onboard FAAM, ARIES (scaled by the Heimann) and TAFTS provide the surface temperature and spectral upwelling and downwelling radiances, respectively. As noted above, measurements of the temperature and the water vapour volume mixing ratio at the aircraft altitude ( $\mathrm{z}$ ) are used to scale the radiosonde atmospheric profiles from Summit. These scaled profiles are then provided as input to LBLRTM and the emission and transmission of the atmosphere between the aircraft and surface are simulated. In the Lambertian case, LBLRTM is also used to simulate downwelling radiances at zenith angles of $0^{\circ}$ and $55^{\circ}$ in order to provide the scaling in equation 10 , requiring knowledge of the atmospheric profile above the aircraft. In this case the adjusted temperature and water vapour profiles from Summit for altitudes above the aircraft flight level were used over their reliable extent (up to $\square 10 \mathrm{~km}$ from the ground) before being merged with the nearest available ERA-I profiles in space and time at higher altitudes.

\section{Results}

\subsection{Emissivity retrievals}

The observed TAFTS nadir and zenith radiances from flight B898 at 14:51 UTC are displayed in Figure 4(a). The gap between 300 and $330 \mathrm{~cm}^{-1}$ separates the range of the LW and SW detectors. The TAFTS noise equivalent spectral radiance is typically less than $1 \mathrm{~mW}$ $\mathrm{m}^{-2} \mathrm{sr}^{-1}\left(\mathrm{~cm}^{-1}\right)^{-1}$ in the $\mathrm{LW}$ and $2 \mathrm{~mW} \mathrm{~m}^{-2} \mathrm{sr}^{-1}\left(\mathrm{~cm}^{-1}\right)^{-1}$ in the $\mathrm{SW}$, with higher values close to the edges of the detector ranges (Figure 4(b)). Figure 4(a) does indicate the presence of a substantial number of negative radiances in the zenith observations. This is a result of relaxed quality controls used to select the zenith scans to be integrated. While viewing in the zenith, an increase in aircraft induced vibrations was seen to cause periodic sampling errors of the TAFTS interferogram resulting in spectral ghosting. Spectral ghosts can be identified by outof-band spectral signals and this is done routinely as part of the TAFTS quality control process, with spectra that show out-of-band residuals greater than the expected noise level 
excluded. In this case, due to the limited number of zenith spectra passing the nominal noise level test we have relaxed the acceptance threshold; this manifests as an increasing negative radiance bias at higher wavenumbers. For this reason we limit our emissivity retrievals to frequencies below $535 \mathrm{~cm}^{-1}$.

In the LW channel, both nadir and zenith radiances follow the Planck function at a temperature around $250 \mathrm{~K}$, indicating that the majority of the emission measured at these wavenumbers originates from close to the aircraft level. Small deviations away from the Planck curve in the nadir view in the LW indicate the presence of micro-windows, which get progressively more transparent with increasing wavenumber. These manifest as dips in the emitted upwelling radiance due to the temperature structure of the atmospheric layer below the aircraft and, in particular, the strong surface temperature inversion. In the SW, this pattern of increasing atmospheric transparency with wavenumber continues to the edge of the 667 $\mathrm{cm}^{-1} \mathrm{CO}_{2}$ band (at around $550 \mathrm{~cm}^{-1}$ ). This can be seen in the nadir view, which has a high sensitivity to surface conditions, but is also clearly apparent in the zenith observations. Here, the temperature and humidity of the layers above the aircraft shape the observed downwelling radiance.

Figures 5(a) and (b) corroborate the inferences made above from the TAFTS observations. Figure 5(a) shows the LBLRTM simulated transmission of the layer between the aircraft and surface using the profile discussed in section 3.2. Figure 5(b) shows the corresponding simulated upward and downward emission of the layer. The most promising regions for the retrieval of surface emissivity show transmittances close to 1 and are located within the TAFTS SW band. Below $300 \mathrm{~cm}^{-1}$, in the LW band, the underlying trend is for transmission to reduce with reducing wavenumber as one moves towards the centre of the water vapour rotation band. The differences between upwelling and downwelling emission are most obvious in the more opaque parts of the LW band and are linked to the temperature structure of the layer, with larger emission upward at the aircraft due to the warmer temperatures at this level compared to near the surface (Figure 2(a)).

Using the simulated transmission and emission, the measured TAFTS nadir and zenith radiances and the retrieved surface temperature, the spectral surface emissivity at nadir is calculated assuming Lambertian reflection using equation 6 and displayed in Figure 6. A cluster of points around an emissivity of 1 is visible, however there are a number of obviously unphysical outliers. These are located in regions of low transmission where the surface signal is small (Figure 5(a)). Hence, a number of steps are employed to sub-sample the data.

The first step is related to the TAFTS data. As noted previously, there is a tendency towards an increasing number of negative zenith radiances at the high wavenumber end of the SW band related to the relaxation of the quality control tests applied to these data. Here we simply discard these negative radiances, which correspond to $2.2 \%$ of the total data points (Figure 7(a)). The second step is linked to the transmission simulated with LBLRTM in the SW band. Here only regions where the simulated transmission is higher than 0.95 are used to retrieve the emissivity, reducing the uncertainty linked to the atmospheric profile. This criterion discards $18.5 \%$ of the measurements (Figure 7(b)). The last step concerns the LW 
range. We use a condition on the Planck function at the surface temperature and at the atmospheric layer temperature (estimated at $250 \mathrm{~K}$ ) to limit our emissivity calculations to the more transparent micro-windows in this band. The condition applied to the nadir observation is:

$$
L_{v, T A F T S}^{\uparrow} \leq B_{v}\left(T_{S}\right)+\left(B_{v}\left(T_{A}\right)-B_{v}\left(T_{S}\right)\right) * \text { limit }
$$

with an empirical 'limit' set at $60 \%$. This effectively means that we are looking at observations close to the Planck emission of the surface. The condition is extended with a lower limit $(20 \%)$ in the SW up to the first wavenumber that satisfies the condition on transmission $\left(363 \mathrm{~cm}^{-1}\right)$, ensuring continuity between the LW and SW conditions. Figure $7(\mathrm{c})$ shows where this condition is met (green dots). The blue and red lines correspond to $B_{v}\left(T_{A}\right)$ and $B_{v}\left(T_{S}\right)$ respectively. $32.1 \%$ of the measurements are discarded. Each condition in panels (a) to (c) is displayed in a specific colour. The corresponding emissivity-frequency distribution obtained with each condition applied sequentially is shown in Figure 7(d). The resulting emissivity distribution gets smaller and tighter as each condition is applied.

The resulting sub-sampled surface emissivity is displayed in Figure 8 . Below $300 \mathrm{~cm}^{-}$ ${ }^{1}$, the retrievals are clustered within the small micro-windows seen in Figure 7(c). The first of these clusters displays a large spread around a mean value of 1.06 . The retrieved emissivity then decreases down to a mean value of 0.86 in the micro-window centred at $220 \mathrm{~cm}^{-1}$ before recovering to a median value close to 0.92 within the $240 \mathrm{~cm}^{-1}$ micro-window. Average emissivity values in each subsequent LW micro-window stay at around 0.95 as the wavenumber increases but the spread in the retrievals also increases. The beginning of the sub-sampled short wave range starts at $330 \mathrm{~cm}^{-1}$, with a median emissivity value of 0.97 . The emissivity stays at 0.95 before reaching unphysical values above 1 between 365 and $385 \mathrm{~cm}^{-}$ ${ }^{1}$. Then, the values show a distinct dip down to 0.91 at $400 \mathrm{~cm}^{-1}$. This feature is relatively pronounced, with the emissivity continuing to vary between 0.90 and 0.93 until it reaches a local minimum (0.89) at around $460 \mathrm{~cm}^{-1}$. Then, an increase up to 1 is observed, which is correlated with an increase of the associated spread. Although there is a significant amount of scatter in the SW retrievals it is noticeably reduced relative to the LW band.

\subsection{Uncertainty analysis}

The estimated percentage error in the retrievals due to instrumental uncertainty is computed using equation 7 and is displayed as a frequency distribution in Figure 9. Most of the retrieved emissivity values (43\%) have measurement induced, or 'direct' uncertainties of less than $5 \%$, with the strongest peak at $2.6 \%$. The lowest percentage uncertainties are $2 \%$ and are linked to the minimum TAFTS measurement uncertainty of about $1 \%$. The errors in excess of $15 \%$ are located in the LW range $\left(v<240 \mathrm{~cm}^{-1}\right)$.

We estimate the effect of uncertainties in the atmospheric profile on the retrieved emissivity $\left(\Delta \boldsymbol{\varepsilon}_{\boldsymbol{v}, \text { ind }}\right)$ using a bootstrap analysis. Excepting the role of surface temperature in exceptionally transparent regions, the water vapour and temperature profiles are the most important parameters in the retrieval of FIR emissivity since most of the absorption in the FIR is due to water vapour (Harries et al., 2008). We computed the retrieved emissivity at 
each wavenumber for more than 1000 cases where the atmospheric profiles of the water vapour and temperature were modified. The error distribution for both parameters was considered to be Gaussian at each pressure level, with a standard deviation of $1.3 \mathrm{~K}$ in temperature and $20.5 \%$ in water vapour mixing ratio, consistent with the differentials between the sonde profile and the FAAM measurements at the same altitude. Perturbations were applied on the whole profile to avoid compensation between levels, and the perturbation was assumed to be coherent, meaning that an increase in temperature was accompanied by an increase in water vapour (essentially minimising variations in relative humidity). Examples of selected perturbations ( \pm 1 and 2 times the standard deviation $\sigma$ ) and their impact on the retrieved emissivity are shown in Figure 10. The impact on the LW emissivity is high (exceeding $20 \%$ for a $1 \sigma$ perturbation in some micro-windows) and generally decreases with increasing wavenumber. Here, reduced water vapour and temperature leads to a lower retrieved emissivity. In contrast, the SW displays much reduced sensitivity with variations of, at most, $3 \%$ reducing to below $1 \%$ above $360 \mathrm{~cm}^{-1}$ for $1 \sigma$ perturbations. Here, reductions in water vapour and temperature lead to an increase in the retrieved emissivity.

\section{Discussion}

As seen in Figures 7 and 8, the Lambertian surface emissivity is only retrieved for particular wavenumbers due to the sub-sampling of the data. Figure 11 displays the median emissivity calculated across $5 \mathrm{~cm}^{-1}$ wide spectral bands in the SW and for each microwindow (numbering 9) in the LW. The spectral behaviour of the emissivity described in section 4.1 becomes more obvious. With the 'almost' regular spectral grid, the median value of the emissivity is 0.95 across the whole spectral range, with values of 0.94 and 0.96 in the LW and SW, respectively. The instrumental, or 'direct' uncertainty is shown in red, the uncertainty resulting from the bootstrap analysis ('indirect') in blue and the combined error (equation 8 ) by the black dash-dot line.

The SW uncertainty computed through the bootstrap analysis is very small (median value of $0.2 \%$ ) since the sub-sampling of data described in section 4.1 implicitly selects wavenumbers with high transmission (Figure 7(b)) where the atmospheric profile has a small impact on the radiance. This contrasts to the LW channel where water vapour absorption has a strong impact. Therefore, the uncertainty in the retrieved emissivity in the LW is greater, reaching values of up to $20 \%$. Similarly, the direct uncertainty on the emissivity is higher in the LW in comparison to the SW with median values of $24.8 \%$ and $4.6 \%$, respectively. Although our results strongly imply that surface emissivity is less than 1.0 across the majority of FIR, combining the indirect and the direct uncertainties associated with the retrievals means that excepting the spectral range between 400 and $480 \mathrm{~cm}^{-1}$ our emissivity estimates cannot be said to be truly distinct from a value of 1.0.

Next we make a comparison of our FIR retrievals with the theoretical data of C14. These describe snow IR spectral emissivity according to two parameters: the fill factor (degree of packing, ranging from 0.1 to 0.7 ) and the effective radius of the snow grains (ranging from 5.2 to $225 \mu \mathrm{m}$ ). The snow emissivity is simulated using the emissivity model described by Hapke (1993) with modified snow optical properties. The modifications are (1) accounting for dense packing effects following the method outlined in Mishchenko (1994) 
and (2) accounting for the rough surface welding effect following Wald (1994). More details can be found in $\mathrm{C} 14$. Across the full range of parameter values and wavelengths considered, the theoretical emissivity takes values from 0.7 to 1 . The data are provided at a spectral resolution which varies from $1 \mathrm{~cm}^{-1}$ to $20 \mathrm{~cm}^{-1}$. The retrieved values are sampled to be consistent with this resolution. We then extract a fill factor and an effective radius that minimises the variance between the theoretical and the retrieved emissivity (weighted and unweighted by the uncertainty). Figures 12(a) (weighted case) and (b) (unweighted case) show the variances as a function of effective radius and fill factor. Clearly, a different effective radius minimises the variance seen for each fill factor: as fill factor increases the effective radius corresponding to the lowest variance also increases. Across the full range of model parameter space, for the weighted case the global minimum in variance is found for a fill factor of 0.2 and an effective radius of $26 \mu \mathrm{m}$. For the unweighted case these values change to 0.3 and $57 \mu \mathrm{m}$. Figure 12(c) provides a comparison of the retrieved emissivity (in black) and the theoretical values corresponding to each of these 'best fits' (in blue). Maximum and minimum theoretical values across the whole parameter space range are shown by the red dashed lines. The resolution of the $\mathrm{C} 14$ data is highlighted by the blue triangles. Both the absolute magnitude and spectral structure in the retrievals and the selected theoretical representations are broadly consistent, with noticeable dips in emissivity between 180 and $230 \mathrm{~cm}^{-1}$ and between 400 and $480 \mathrm{~cm}^{-1}$. The weighted version shows a better match in the SW as expected since the uncertainties are smaller in this region.

Can the same model parameters also provide an adequate fit to near simultaneous measurements of the emissivity in the MIR? To answer this question we extract emissivity retrievals from ARIES, derived over the spectral range 760-1240 $\mathrm{cm}^{-1}$ using the approach of Newman et al. (2005) (see also Thelen et al., 2009). We select the same sequence of observations used to derive the surface temperature used in the TAFTS retrievals, resulting in a total of 119 retrieved emissivity spectra. Figure 13 shows the mean of these retrievals as a function of wavenumber (as red crosses), binned to the resolution of C14. The shaded grey area is indicative of the range in each of these binned values over the 119 spectra and is used as a proxy for the uncertainty in the retrievals.

The emissivity measured by ARIES varies between 0.98 and 1 , with a mean value of 0.99 . The spectral shape is quite distinctive, emissivity increasing from $760-950 \mathrm{~cm}^{-1}$, reducing from $950-1050 \mathrm{~cm}^{-1}$ and then increasing with increasing wavenumber to $1240 \mathrm{~cm}^{-1}$. Minimising variance as before, the closest simulations from $\mathrm{C} 14$ are displayed as red solid lines with, in Fig. 13(a), no weighting applied and in (b) uncertainty weighting applied. The value of the corresponding effective radii is of the same order as those retrieved from TAFTS ( $37 \mu \mathrm{m}$ and $26 \mu \mathrm{m}$ for the weighted and unweighted cases, respectively) but the fill factor increases ( 0.6 and 0.5 , respectively). Moreover, it is obvious that neither simulation closely mimics the behavior in the FIR.

Performing equivalent minimisations over the FIR and MIR simultaneously results in the black curves in Fig. 13(a) and (b). Because of the much smaller uncertainties assumed for ARIES the addition of the FIR information makes little difference to the best match when the variances are weighted (compare the red and black curves in Fig. 13(b)). In contrast, when the variances are unweighted by uncertainties the simulation giving the closest fit shows 
much more consistency with the spectral variations seen in the FIR but has emissivities which are systematically too low when compared to the MIR retrievals. Essentially, if the surface is assumed to be purely snow, there is no representation in the database that can simultaneously match the retrieved MIR and FIR emissivities within uncertainties.

An improved match to the observations is seen using a mixture of uncovered ice and snow, or crusted snow (e.g. Hori et al., 2006). Ice emissivity displays a distinct minimum at $200 \mathrm{~cm}^{-1}$, a feature clearly seen in the TAFTS retrieval. The green dashed line in Fig. 13(a) shows the simulated emissivity for a weighted mixture of ice (45\%) and snow (55\%). Ice emissivity is calculated from the Fresnel equations (Huang et al., 2016) while a snow effective radius of $14.4 \mu \mathrm{m}$ and fill factor of 0.3 minimises the variance between the simulations and retrievals. Although the comparison is enhanced, including an improved match to the observed spectral variation in the MIR, we can conclude that there is no solution that matches the retrievals across the IR. This indicates the need for more targeted measurements (including the relevant snow/ice parameters) and an improved modelling approach.

\section{Conclusion}

In this study we have used aircraft observations from the CIRCCREX-COSMICS measurement campaign in March 2015 to retrieve the far-infrared surface emissivity over a limited region of the Greenland ice sheet. We have described the retrieval process, using radiance observations from the TAFTS and ARIES instruments along with ancillary information of the atmospheric state, under the assumption of Lambertian surface reflectance. The FIR retrieval requires the simulation of the transmission and atmospheric emission of the layer between the aircraft and the surface and a correction of the downwelling radiance above the aircraft, as well as an independent measurement of the surface temperature. As such, the atmospheric state must be known with a reasonable level of certainty: a condition that becomes more important for wavenumbers where the opacity of the atmospheric layer between the ground and aircraft increases.

The emissivity retrieved using the full range of TAFTS observations is very noisy, primarily at wavenumbers $<388 \mathrm{~cm}^{-1}$ due to atmospheric contamination and, at detector edges, instrument noise. Therefore a method has been developed to sub-sample the data using constraints on the simulated transmission and the TAFTS measurements themselves. After performing this data selection, the retrieved emissivity shows a smoother variation, particularly between $390-510 \mathrm{~cm}^{-1}$, and the values are physically plausible, given uncertainties associated with the measurements and our knowledge of the atmospheric state at the time of the observations. Overall our results indicate that, as suggested by recent work, snow FIR emissivity is lower than 1.0.

Comparing the FIR results with a recently developed theoretical database shows broad agreement, the retrievals showing spectral features consistent with a small grain sized, loosely packed snow cover. However, in extending the comparison to include the MIR using emissivity retrievals from ARIES, we find that no single theoretical representation for a purely snow surface is able to capture the observed behaviour. Combining simulations for 
snow and ice, characteristic of 'crusted snow' allow us to obtain a closer match, including greater consistency with the spectral features seen in the FIR and MIR. The agreement between the retrievals and theoretical representation does however still lie outside of our estimated measurement uncertainties for much of the observed spectral range.

Our results support the hypothesis that the assumption of a constant surface emissivity of 1 over the FIR made in the majority of current climate and Earth System Models (ESMs) will lead to an overestimation in upwelling infrared surface emission over snow covered surfaces. This would be expected to lead to biases in both the surface energy budget and, in cloud-free conditions, the outgoing longwave radiation. The results also highlight the need for both improvements in the modelling of surface emissivity and additional measurement campaigns in order to have a more extensive and better constrained set of FIR/MIR emissivity estimates encompassing a range of different snow conditions. For example, physical in-situ sampling can provide information on characteristics such as the snow density and contact temperature (Hall et al., 2008), while near-infrared measurements can be exploited to estimate snow grain size through a variety of different methods (Gallet et al., 2009, Nolin, 2010). When taken in concert with FIR and MIR radiance measurements these types of observations could be used to perform a much more complete evaluation of recently developed models of IR snow emissivity (Huang et al., 2016) designed for incorporation in the next generation of ESMs.

\section{Acknowledgments}

The CIRCCREX measurement campaign was supported by NERC-grant (grant $\mathrm{NE} / \mathrm{K} 015133 / 1$ ). The subsequent analysis was supported by the NERC funded International Consortium for the Exploitation of Infrared Measurements of PolAr ClimaTe (ICE-IMPACT) project (grant NE/N01376X/1) and by the National Centre for Earth Observation, UK. The radiosonde data were obtained from the ICECAPS project funded by the U.S. National Science Foundation (PLR 1303879). The MODIS level 1b data were acquired from the level1 and Atmospheric Archive and Distribution System (LAADS) Distributed Active Archive Center (DAAC), located in the Greenbelt, Maryland (https://ladsweb.nascom.nasa.gov). ECMWF data were acquired from http://www.ecmwf.int/en/research/climate-reanalysis/erainterim. The FAAM BAe146-301 is jointly funded by the Met Office and the Natural Environment Research Council. We also acknowledge Jennifer Brooke from the Met Office, the technical and pre/post-flight assistance of the Avalon ground crew and Air-Task flight planning during detachment. We are also grateful to the British Oxygen company for the delivery of liquid helium to Iceland when no local source could be found and finally thanks to the Keflavik airport operations crew working for South Air Iceland for support and accommodating our ground operation area.

\section{References}

Allen, G.; Illingworth, S. M.; O'Shea, S. J.; Newman, S.; Vance, A.; Bauguitte, S. J.-B.; Marenco, F.; Kent, J.; Bower, K.; Gallagher, M. W.; Muller, J.; Percival, C. J.;

Harlow, thermodynamic
C.; Lee, J. \& Taylor, J. P. (2014). Atmospheric composition and retrievals from the ARIES airborne TIR-FTS system - 
Part 2: Validation and results from aircraft campaigns, Atmospheric Measurement Techniques, 7, 4401-4416

Canas, T. A.; Murray, J. E. \& Harries, J. E., Haigh, J. D. (1997). Tropospheric airborne Fourier transform spectrometer (TAFTS), Satellite Remote Sensing of Clouds and the Atmosphere II, 3220, 91-102

Chen, X.; Huang, X. \& Flanner, M. G. (2014). Sensitivity of modeled far-IR radiation budgets in polar continents to treatments of snow surface and ice cloud radiative properties, Geophysical Research Letters, 41, 6530-6537

Clough, S.; Shephard, M.; Mlawer, E.; Delamere, J.; Iacono, M.; Cady-Pereira, K.; Boukabara, S. \& Brown, P. (2005). Atmospheric radiative transfer modeling: a summary of the AER codes, Journal of Quantitative Spectroscopy and Radiative Transfer, 91, $233-244$

Cox, C. V.; Murray, J. E.; Taylor, J. P.; Green, P. D.; Pickering, J. C.; Harries, J. E. \& Last, A. E. (2007). Clear-sky far-infrared measurements observed with TAFTS during the EAQUATE campaign, September 2004, Quarterly Journal of the Royal Meteorological Society, 133, 273-283

Cox, C. V.; Harries, J. E.; Taylor, J. P.; Green, P. D.; Baran, A. J.; Pickering, J. C.; Last, A. E. \& Murray, J. (2010). Measurement and simulation of mid- and far-infrared spectra in the presence of cirrus, Quarterly Journal of the Royal Meteorological Society, John Wiley \& Sons, Ltd., 136, 718-739

Dee, D. P.; Uppala, S. M.; Simmons, A. J.; Berrisford, P.; Poli, P.; Kobayashi, S.; Andrae, U.; Balmaseda, M. A.; Balsamo, G.; Bauer, P.; Bechtold, P.; Beljaars, A. C. M.; van de Berg, L.; Bidlot, J.; Bormann, N.; Delsol, C.; Dragani, R.; Fuentes, M.; Geer, A. J.; Haimberger, L.; Healy, S. B.; Hersbach, H.; Hólm, E. V.; Isaksen, L.; Kållberg, P.; Köhler, M.; Matricardi, M.; McNally, A. P.; Monge-Sanz, B. M.; Morcrette, J.-J.; Park, B.-K.; Peubey, C.; de Rosnay, P.; Tavolato, C.; Thépaut, J.-N. \& Vitart, F. (2011). The ERA-Interim reanalysis: configuration and performance of the data assimilation system, Quarterly Journal of the Royal Meteorological Society, John Wiley \& Sons, Ltd., 137, 553-597

Feldman, D. R.; Collins, W. D.; Pincus, R.; Huang, X. \& Chen, X. (2014). Far-infrared surface emissivity and climate, Proceedings of the National Academy of Science, 111, 16297-16302

Fox, C.; Green, P. D.; Pickering, J. C. \& Humpage, N. (2015). Analysis of far-infrared spectral radiance observations of the water vapor continuum in the Arctic, JQSRT, $155,57-65$

Gallet, J.-C.; Domine, F.; Zender, C. S. \& Picard, G. (2009). Measurement of the specific surface area of snow using infrared reflectance in an integrating sphere at 1310 and $1550 \mathrm{~nm}$, The Cryosphere, 3, 167-182

GLOBE Task Team and others (Hastings, David A., Paula K. Dunbar, Gerald M. Elphingstone, 

Holland,

Mark Bootz, Hiroshi Murakami, Hiroshi Maruyama, Hiroshi Masaharu, Peter John Payne, Nevin A. Bryant, Thomas L. Logan, J.-P. Muller, Gunter Schreier, and John

S. MacDonald), (1999). The Global Land One-kilometer Base Elevation (GLOBE)

Digital Elevation Model, Version 1.0. National Oceanic and Atmospheric Administration,

National Geophysical Data Center, 325 Broadway, Boulder, Colorado 80303, U.S.A.

Digital data base on the World Wide Web (URL:

http://www.ngdc.noaa.gov/mgg/topo/globe.html) and CDROMs.

Green, P. D.; Newman, S. M.; Beeby, R. J.; Murray, J. E.; Pickering, J. C. \& Harries, J. E. (2012). Recent advances in measurement of the water vapour continuum in the farinfrared spectral region, Philosophical Transactions of the Royal Society of London Series A, 2012, 370, 2637-2655

Guedj, S.; Karbou, F.; Rabier, F. \& Bouchard, A. (2010). Toward a Better Modeling of Surface Emissivity to Improve AMSU Data Assimilation Over Antarctica, IEEE Transactions on Geoscience and Remote Sensing, 48, 1976-1985

Hall, D. K.; Box, J. E.; Casey, K. A.; Hook, S. J.; Shuman, C. A. \& Steffen, K. (2008). Comparison of satellite-derived and in-situ observations of ice and snow surface temperatures over Greenland, Remote Sensing of Environment, 112, 3739 - 3749

Hapke, B. (1993). Theory of reflectance and emittance spectroscopy, Topics in Remote Sensing, Cambridge, UK: Cambridge University Press

Harlow, R. C., Millimeter Microwave Emissivities and Effective Temperatures of SnowCovered Surfaces: Evidence for Lambertian Surface Scattering (2009). IEEE Transactions on Geoscience and Remote Sensing, 47, 1957-1970

Harries, J.; Carli, B.; Rizzi, R.; Serio, C.; Mlynczak, M.; Palchetti, L.; Maestri, T.; Brindley, H. \& Masiello, G. (2008). The Far-infrared Earth, Reviews of Geophysics, 46, RG4004

Highwood, E. J.; Haywood, J. M.; Silverstone, M. D.; Newman, S. M. \& Taylor, J. P. (2003). aircraft Radiative properties and direct effect of Saharan dust measured by the C-130 during Saharan Dust Experiment (SHADE): 2. Terrestrial spectrum, Journal of Geophysical Research: Atmospheres, 108, 8578

Hori, M.; Aoki, T.; Tanikawa, T.; Motoyoshi, H.; Hachikubo, A.; Sugiura, K.; Yasunari, T. J.; $\quad$ Eide, H.; Storvold, R.; Nakajima, Y. \& Takahashi, F. (2006). In-situ measured spectral directional emissivity of snow and ice in the 8-14 $\mu \mathrm{m}$ atmospheric window, Remote Sensing of Environment, 100, 486 - 502

Huang, X.; Chen, X.; Zhou, D. K. \& Liu, X. (2016). An Observationally Based Global Bandby-Band Surface Emissivity Dataset for Climate and Weather Simulations, Journal of the Atmospheric Sciences, 73, 3541-3555 
Knuteson, R.; Best, F.; DeSlover, D.; Osborne, B.; Revercomb, H. \& Sr., W. S. (2004).

Infrared land surface remote sensing using high spectral resolution aircraft observations, Advances in Space Research, 33, 1114 - 1119

Li, Z.-L.; Wu, H.; Wang, N.; Qiu, S.; Sobrino, J. A.; Wan, Z.; Tang, B.-H. \& Yan, G. (2013).

Land surface emissivity retrieval from satellite data, International Journal of

Remote Sensing, 34, 3084-3127

Mätzler, C. (2005). On the Determination of Surface Emissivity From Satellite Observations, IEEE Geoscience and Remote Sensing Letters, 2, 160-163

Mishchenko, M. I. (1994). Asymmetry parameters of the phase function for densely packed scattering grains, Journal of Quantitative Spectroscopy and Radiative Transfer, 52, 95 $-110$

Newman, S. M.; Smith, J. A.; Glew, M. D.; Rogers, S. M. \& Taylor, J. P. (2005).

Temperature and salinity dependence of sea surface emissivity in the thermal infrared, Quarterly Journal of the Royal Meteorological Society, John Wiley \& Sons, Ltd., 131, 2539-2557

Newman, S. M.; Larar, A. M.; Smith, W. L.; Ptashnik, I. V.; Jones, R. L.; Mead, M. I.;

Revercomb, H.; Tobin, D. C.; Taylor, J. K. \& Taylor, J. P. (2012). The Joint

Airborne IASI Validation Experiment: An evaluation of instrument and algorithms, Journal of Quantitative Spectroscopy and Radiative Transfer, 113, 1372 1390

Nolin, A. W. (2010). Recent advances in remote sensing of seasonal snow, Journal of Glaciology, 56, 1141-1150

Rothman, L. S.; Gordon, I. E.; Babikov, Y.; Barbe, A.; Chris Benner, D.; Bernath, P. F.; Birk, M.; Bizzocchi, L.; Boudon, V.; Brown, L. R.; Campargue, A.; Chance, K.; Cohen, E. A.; Coudert, L. H.; Devi, V. M.; Drouin, B. J.; Fayt, A.; Flaud, J.-M.; Gamache, R. R.; Harrison, J. J.; Hartmann, J.-M.; Hill, C.; Hodges, J. T.; Jacquemart, D.; Jolly, A.; Lamouroux, J.; Le Roy, R. J.; Li, G.; Long, D. A.; Lyulin, O. M.; Mackie, C. J.; Massie, S. T.; Mikhailenko, S.; Müller, H. S. P.; Naumenko, O. V.; Nikitin, A. V.; Orphal, J.; Perevalov, V.; Perrin, A.; Polovtseva, E. R.; Richard, C.; Smith, M. A. H.; Starikova, E.; Sung, K.; Tashkun, S.; Tennyson, J.; Toon, G. C.; Tyuterev, V. G. \& Wagner, G. (2013). The HITRAN2012 molecular spectroscopic database, Journal of Quantitative Spectroscopy and Radiative Transfer, 130, 4-50

Shupe, M. D.; Turner, D. D.; Walden, V. P.; Bennartz, R.; Cadeddu, M. P.; Castellani, B. B.; Cox, Rowe,

C. J.; Hudak, D. R.; Kulie, M. S.; Miller, N. B.; Ryan R. Neely, I.; Neff, W. D. \&

P. M. (2013). High and Dry: New Observations of Tropospheric and Cloud Properties above the Greenland Ice Sheet, Bulletin of the American Meteorological Society, 94, 169-186 
Thelen, J.-C.; Havemann, S.; Newman, S. M. \& Taylor, J. P. (2009). Hyperspectral retrieval of land surface emissivities using ARIES, Quarterly Journal of the Royal Meteorological Society, John Wiley \& Sons, Ltd., 135, 2110-2124

Thome, K.; Biggar, S. \& Takashima, T. (1999). Algorithm theoretical basis document for ASTER level 2B1, surface radiance, and ASTER level 2B5, surface reflectance, Contract NAS5-31 717

Vance, A. K.; Abel, S. J.; Cotton, R. J. \& Woolley, A. M. (2015). Performance of WVSS-II hygrometers on the FAAM research aircraft, Atmospheric Measurement Techniques, $8,1617-1625$

Wald, A. E. (1994). Modeling thermal infrared (2-14 $\mu \mathrm{m})$ reflectance spectra of frost and snow, Journal of Geophysical Research: Solid Earth, 99, 24241-24250

Wilson, S. H. S.; Atkinson, N. C. \& Smith, J. A. (1999). The Development of an Airborne Infrared Interferometer for Meteorological Sounding Studies, Journal of Atmospheric and Oceanic Technology, 16, 1912

Figure 1: Panel A: Surface elevation (in $\mathrm{m}$ ) from the Global Land One-kilometer Base Elevation (GLOBE task team, 1999) with the CIRCCREX B898 flight path originating from the Keflavík International Airport (Iceland) and overflying Greenland shown by the white track. Panel B: main: $11 \mu \mathrm{m}$ brightness temperature as recorded by the MODerate Imaging Spectroradiometer (MODIS) on TERRA at 13:20 UTC on the $19^{\text {th }}$ March with the B898 flight track overplotted in black. The Summit station is represented by the open white square. Each black star along the B898 flight track indicates a drop sonde release. Inset: Altitude of the flight track (in black) with respect to the ground elevation (in red) over the range bounded by the white dots. The black vertical lines denote the start and end of the TAFTS measurement sequence used to derive the emissivities reported in this study.

Figure 2: Temperature (a) and water vapour (b) profiles between the ground and $630 \mathrm{mb}$ from Summit radiosonde in black. The red squares correspond to the measurements made on board the FAAM aircraft at the time of the TAFTS measurements, as described in the text, with the uncertainties smaller than the size of the box.

Figure 3: Flowchart of the surface emissivity retrieval in the study.

Figure 4: (a) TAFTS nadir and zenith radiance observations (in black and in red respectively) during B898. The Planck function computed at the mean atmospheric temperature measured by the Rosemount de-iced temperature sensor onboard FAAM is displayed in blue. The gap at $300 \mathrm{~cm}^{-1}$ corresponds to the separation between the LW range $\left(80-300 \mathrm{~cm}^{-1}\right)$ and the SW range $\left(330-580 \mathrm{~cm}^{-1}\right)$. (b) Noise Equivalent Spectral Radiance (NESR) in TAFTS nadir data in the LW and SW ranges.

Figure 5: (a) Simulated transmission of the layer between the surface and FAAM aircraft. (b) Simulated emitted radiance of the atmospheric layer between the surface and FAAM aircraft. In black is the upwelling nadir emission, where the observer is at the aircraft level, and in red is the downwelling zenith emission, where the observer is at ground level.

Figure 6: First retrieval of the raw emissivity. Although a trend is visible, high noise levels are present, particularly in the LW, implying data sub-sampling is required.

Figure 7: Methods used to sub-sample the data. (a) Negative zenith radiances observed by TAFTS (highlighted in red) are removed. (b) Only the regions where the simulated layer transmission is higher than 0.95 (highlighted in blue) in the short wave range are used. (c) A condition regarding the long wave range nadir radiance and the Planck functions using the surface and atmospheric temperatures is applied which selects the points in green. (d) Histogram of each emissivity cases with the appropriate condition applied sequentially.

Figure 8: Retrieved sub-sampled emissivity using the points selected in Figure 7. In the LW range, the clustering of crosses corresponds to each micro-windows isolated by the selection process. 
Figure 9: Distribution of the direct emissivity uncertainty (for the sub-sampled data). Most of the uncertainties fall below $5 \%$, with a peak at $2.6 \%$.

Figure 10: (a) Temperature profiles for various perturbations $( \pm 1 \sigma, \pm 2 \sigma)$ used in bootstrap analysis in colour with the original temperature profile in black. (b) Corresponding VMR profiles. (c) Relative variation in retrieved emissivity colour-coded according to the perturbations in (a) and (b). Note that the range in relative variation is different between the SW (RH scale) and the LW (LH scale), with perturbations exerting a much larger impact in the LW.

Figure 11: Final retrieved emissivity (black crosses). In the SW range, the median value across $5 \mathrm{~cm}^{-1}$ width bins is displayed whereas in the LW the crosses correspond to each of the 8 micro-windows selected by the data subsampling. The dash-dot lines represent estimates of the corresponding 'direct' uncertainty (red), 'indirect' uncertainty, as derived from the bootstrap analysis (blue) and the combination of the two (black).

Figure 12: (a) Weighted (solid line) variances between the retrieved spectral emissivity and the theoretical estimates computed for each fill factor along the effective radius. Vertical lines show the position of the minimum variance for each fill factor. (b) Same as (a), but for unweighted (dashed line) variances. (c) Emissivity retrieved using TAFTS data (black dots) binned to the $\mathrm{C} 14$ spectral grid (blue triangles) and closest simulated emissivity from $\mathrm{C} 14$ dataset for the weighted variance (blue solid line) and for the unweighted variance (blue dashed line). The red dashed lines show the maximum and minimum emissivities contained in the C14 dataset.

Figure 13: Retrieved emissivities from TAFTS (blue triangles) and ARIES (red crosses). Grey shading indicates the estimated uncertainties in the retrievals. Corresponding 'best-fit' simulations for a pure snow surface are displayed as solid lines. (a) Minimisations obtained without uncertainty weighting. (b) Weighting included. A simulation mixing ice and snow is displayed with a dashed green line in (a). The numbers in brackets indicate snow fill factor and effective radius for the appropriate best fit simulation.

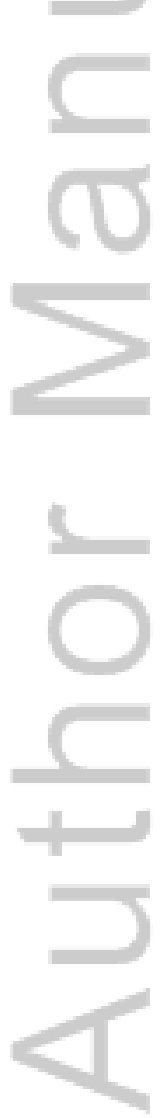




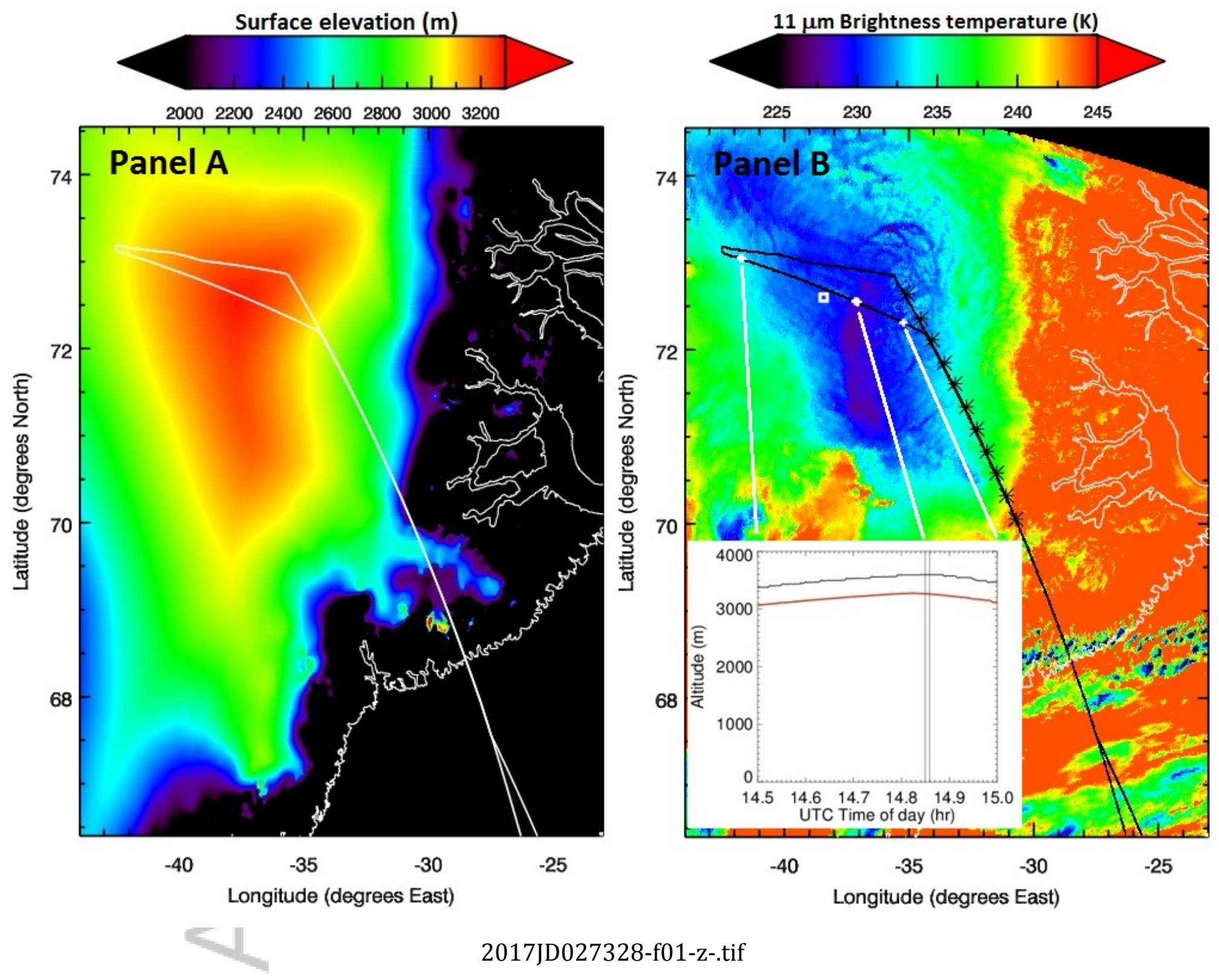

This article is protected by copyright. All rights reserved. 


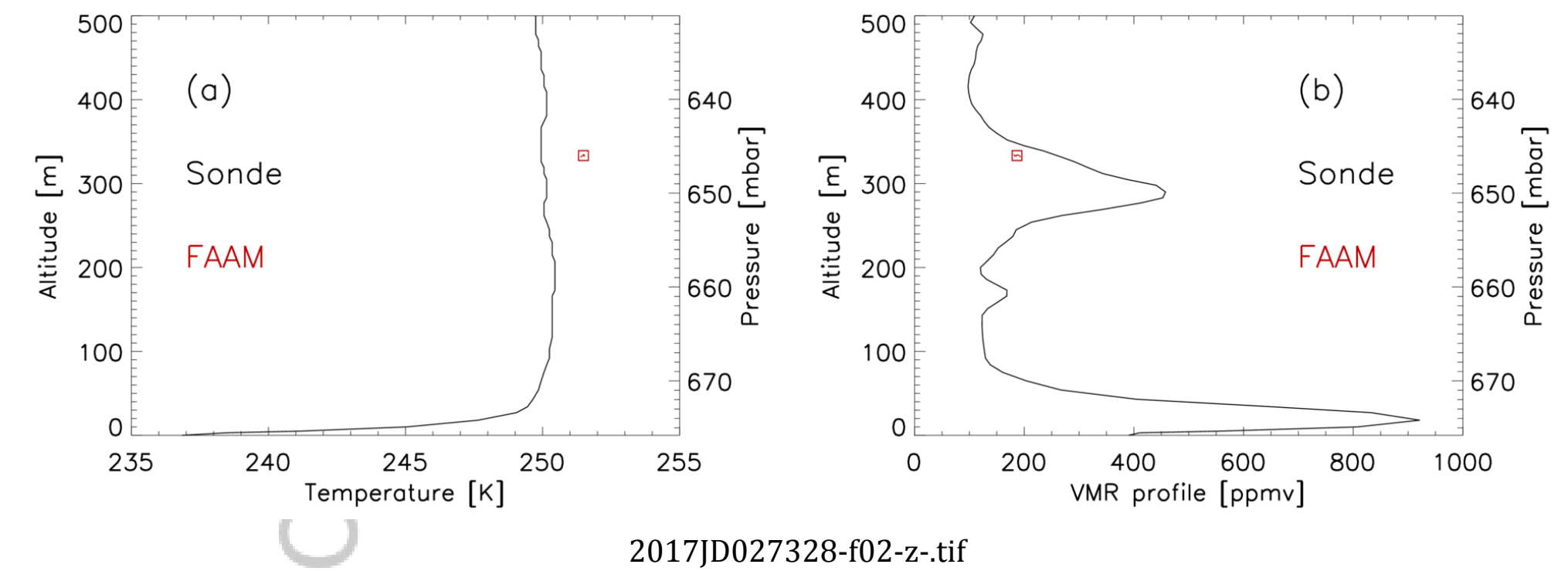

This article is protected by copyright. All rights reserved. 


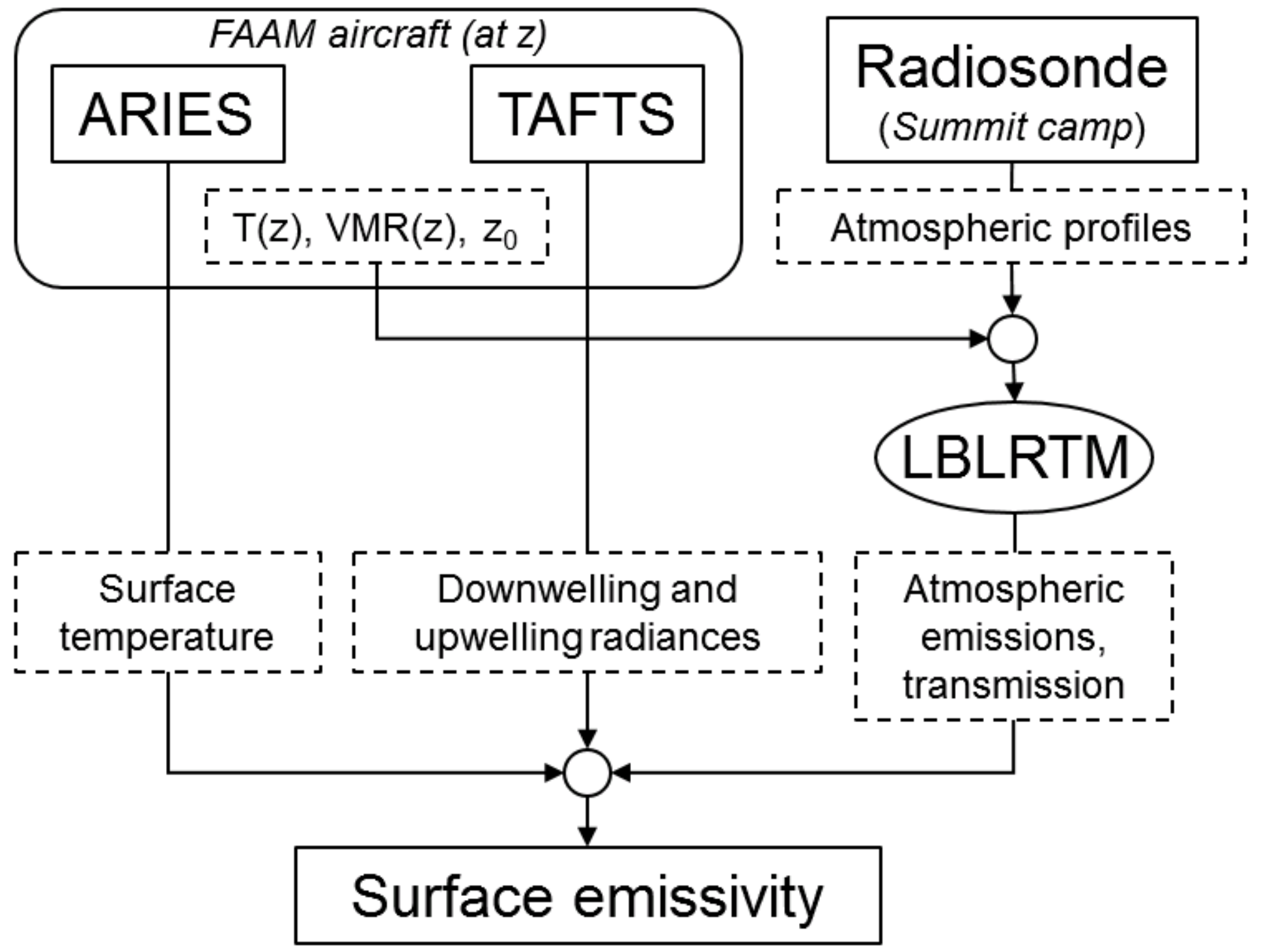

2017JD027328-f03-z-.tif 

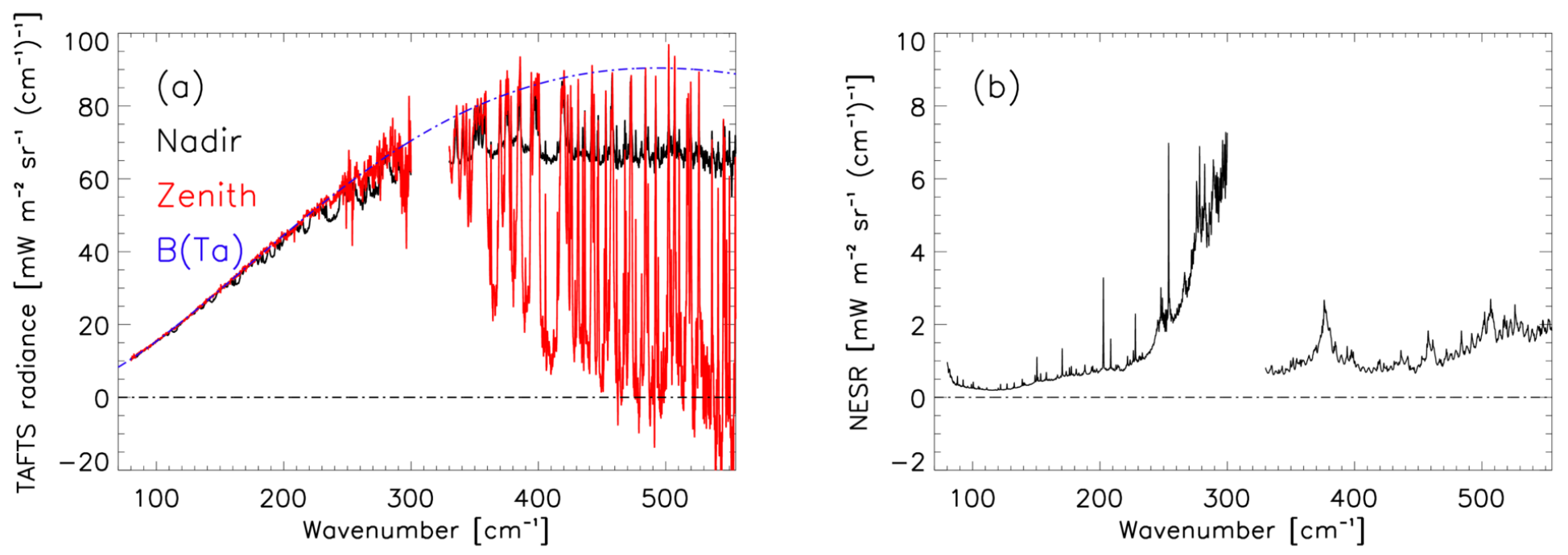

2017JD027328-f04-z-.tif

This article is protected by copyright. All rights reserved. 

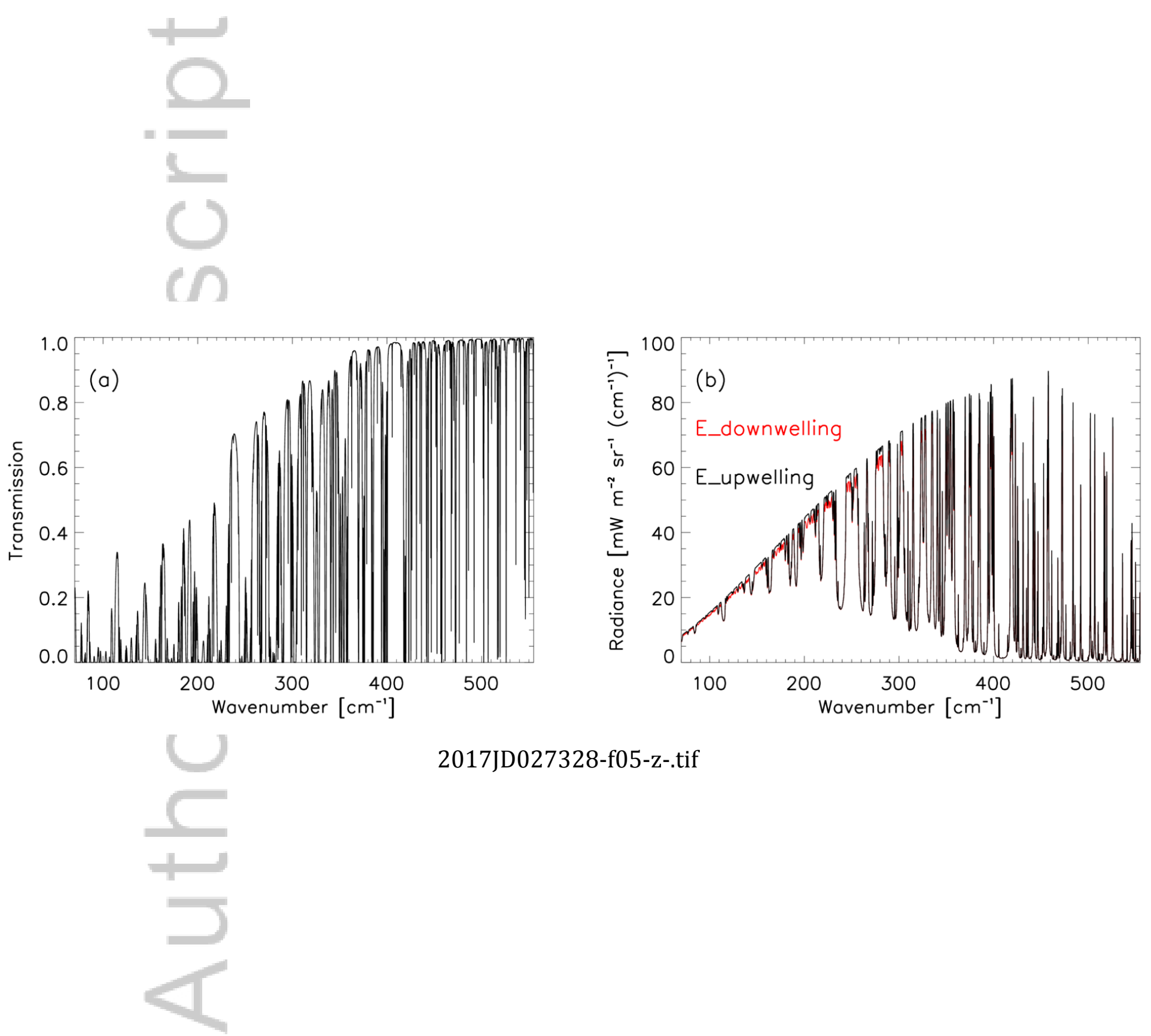

2017JD027328-f05-z-.tif

This article is protected by copyright. All rights reserved. 


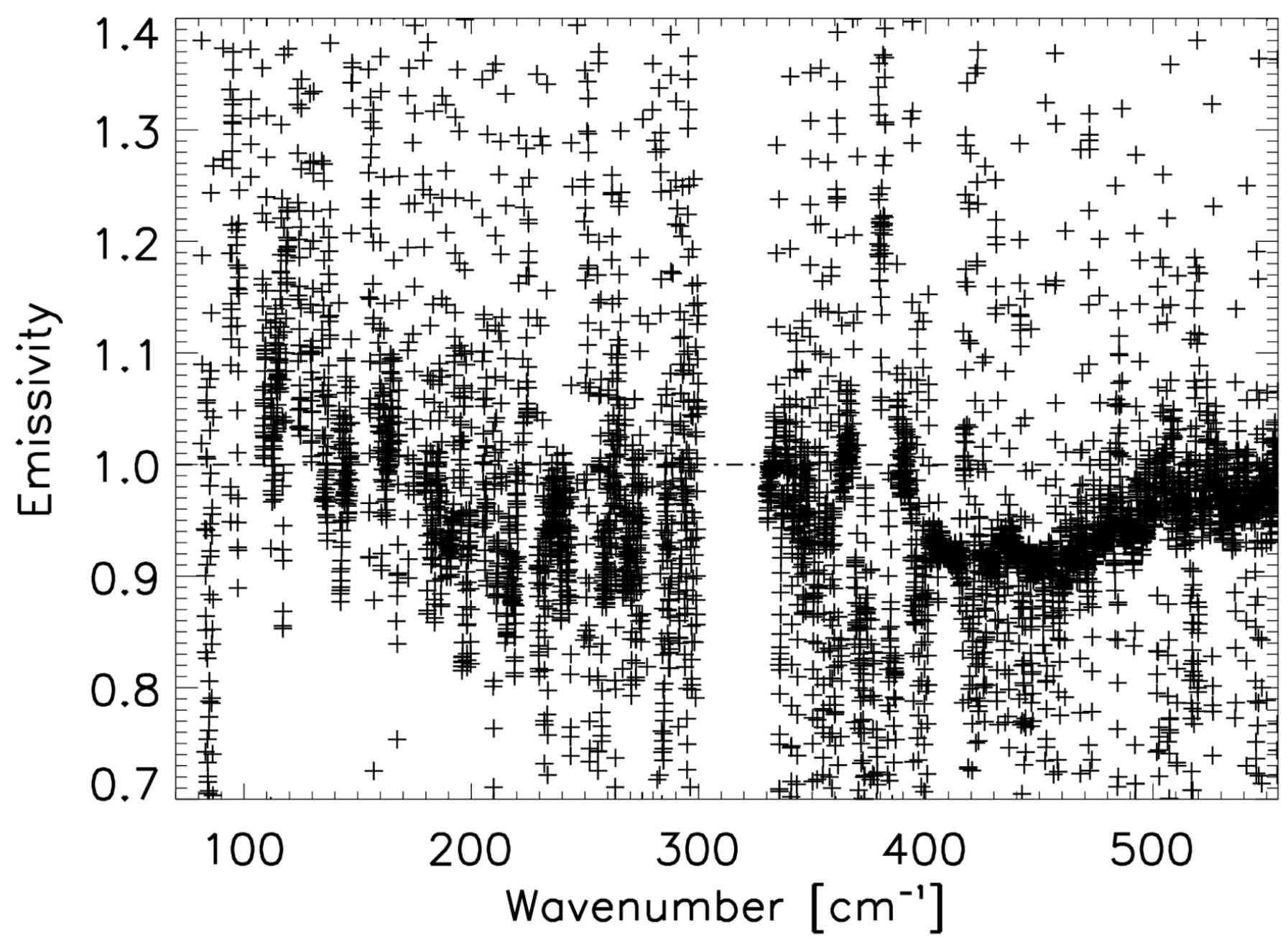

2017JD027328-f06-z-.tif 

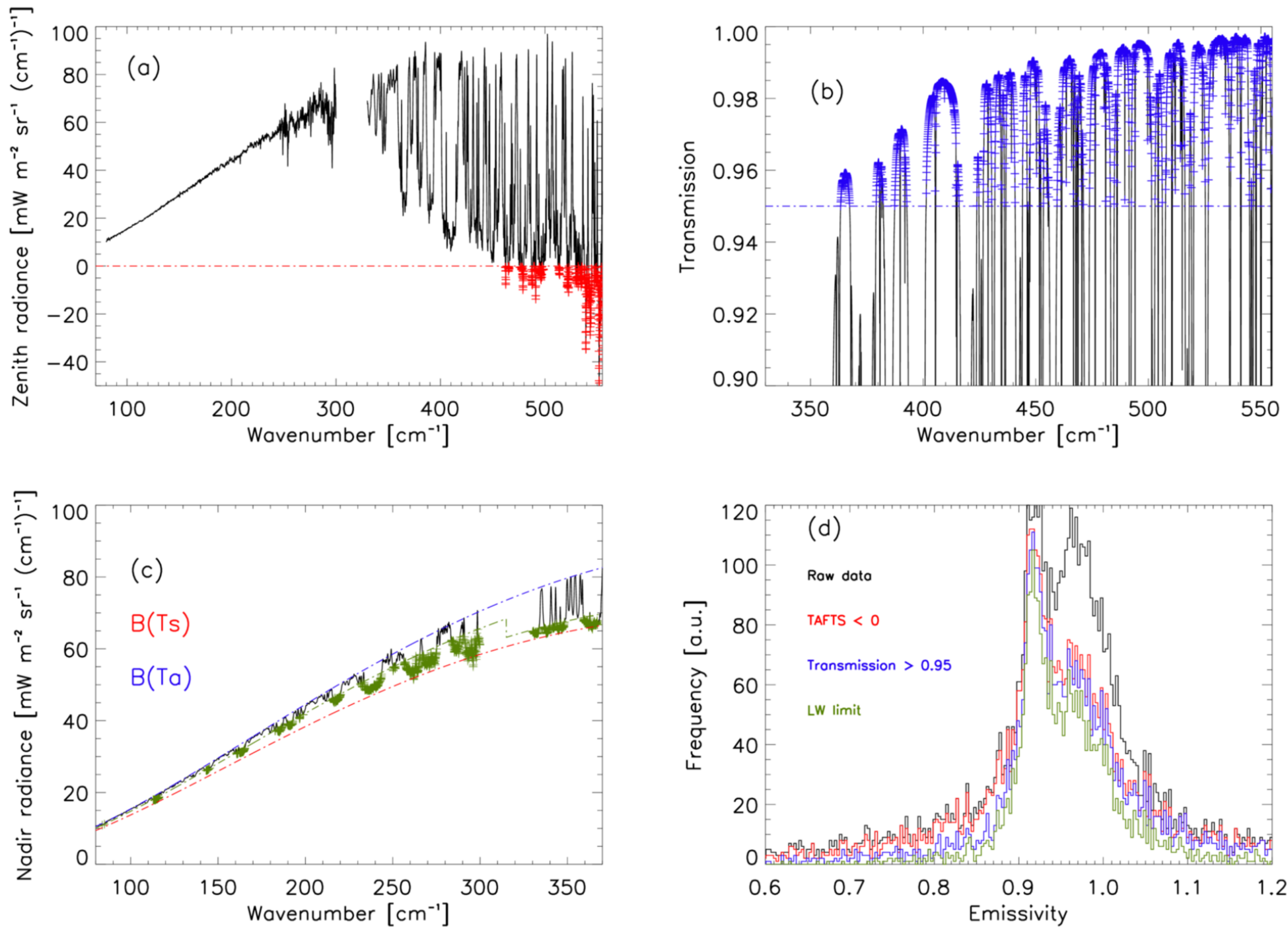

2017JD027328-f07-z-.tif

This article is protected by copyright. All rights reserved. 


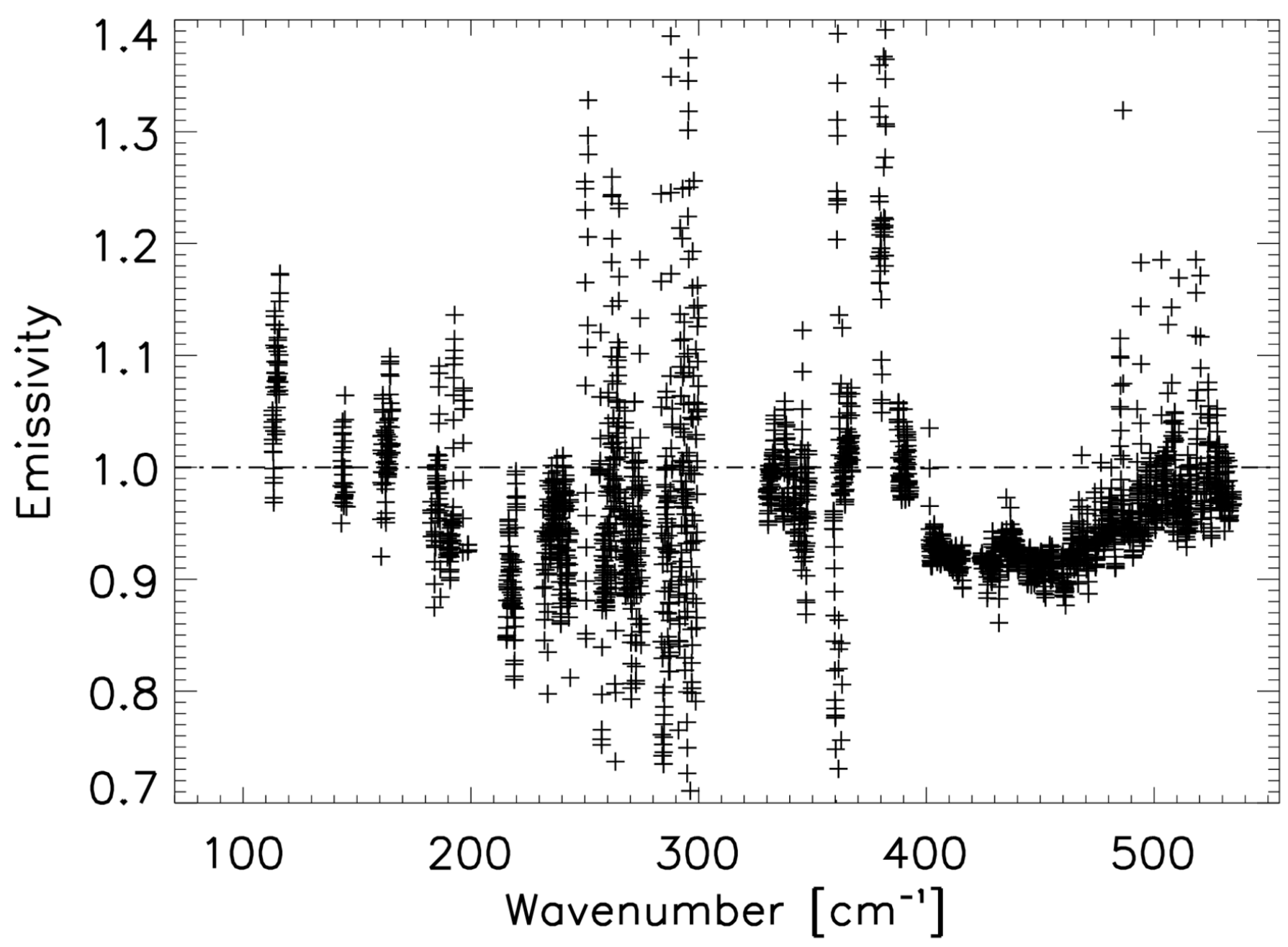

2017JD027328-f08-z-.tif 


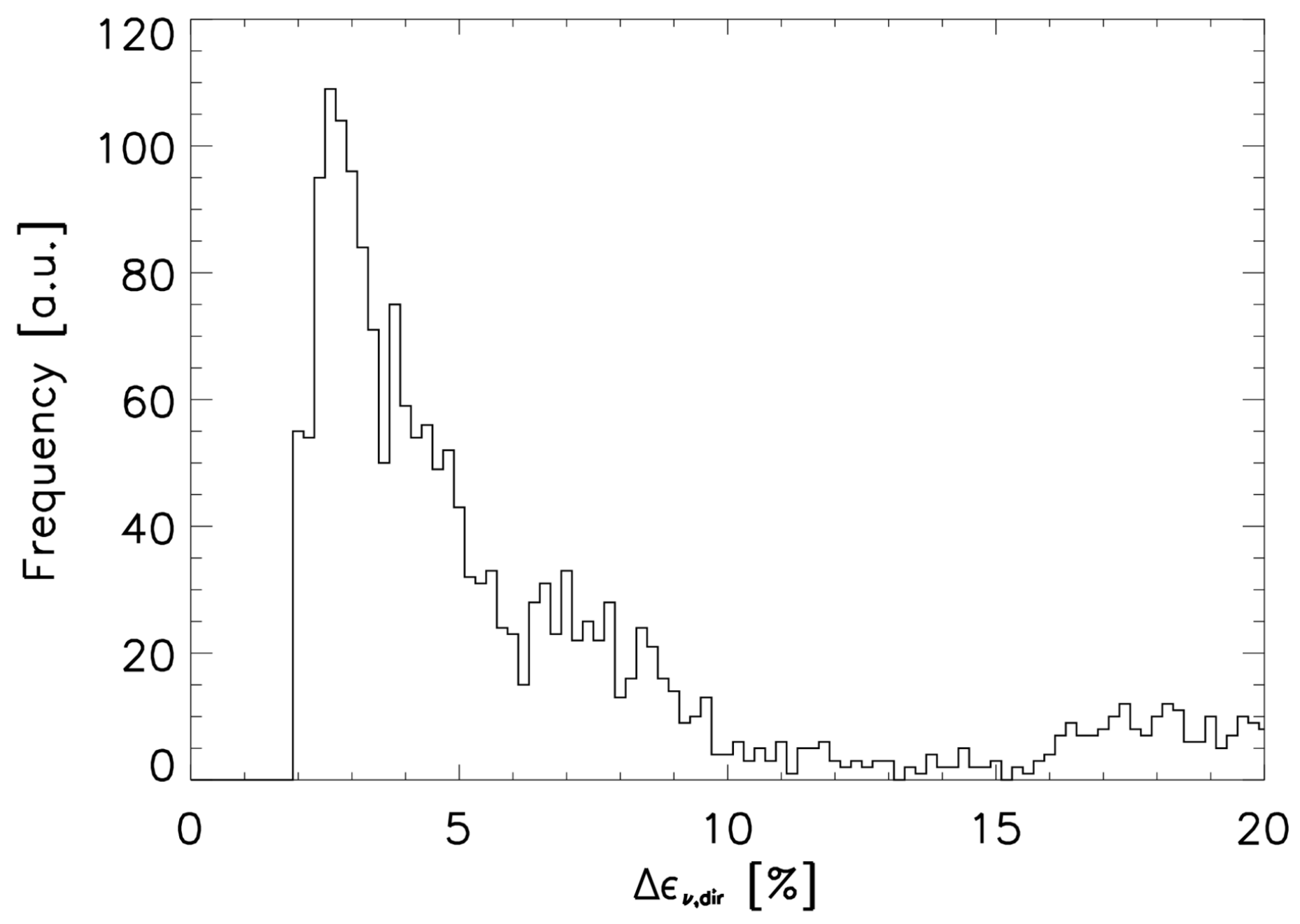

2017JD027328-f09-z-.tif 

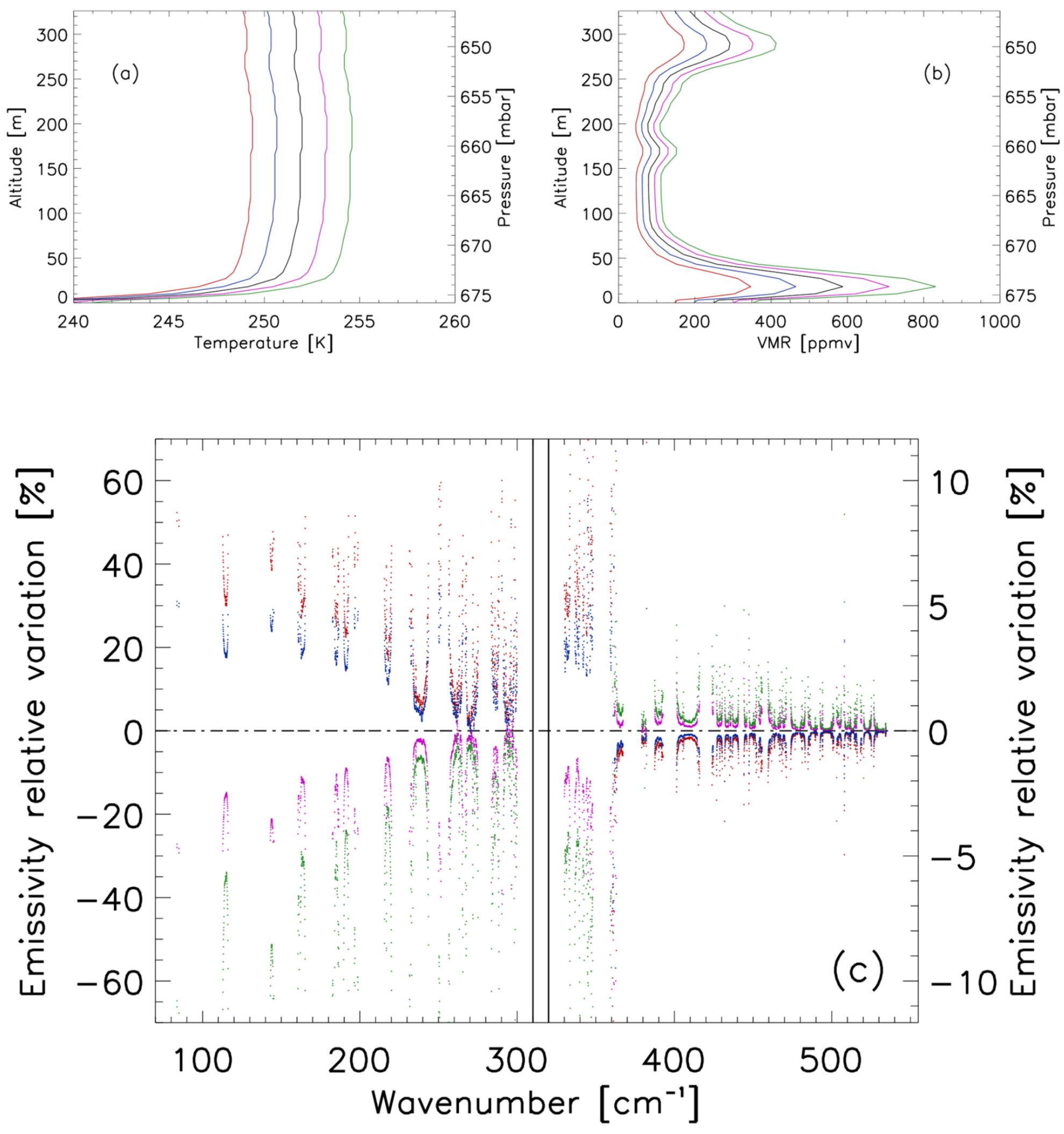

2017JD027328-f10-z-.tif 


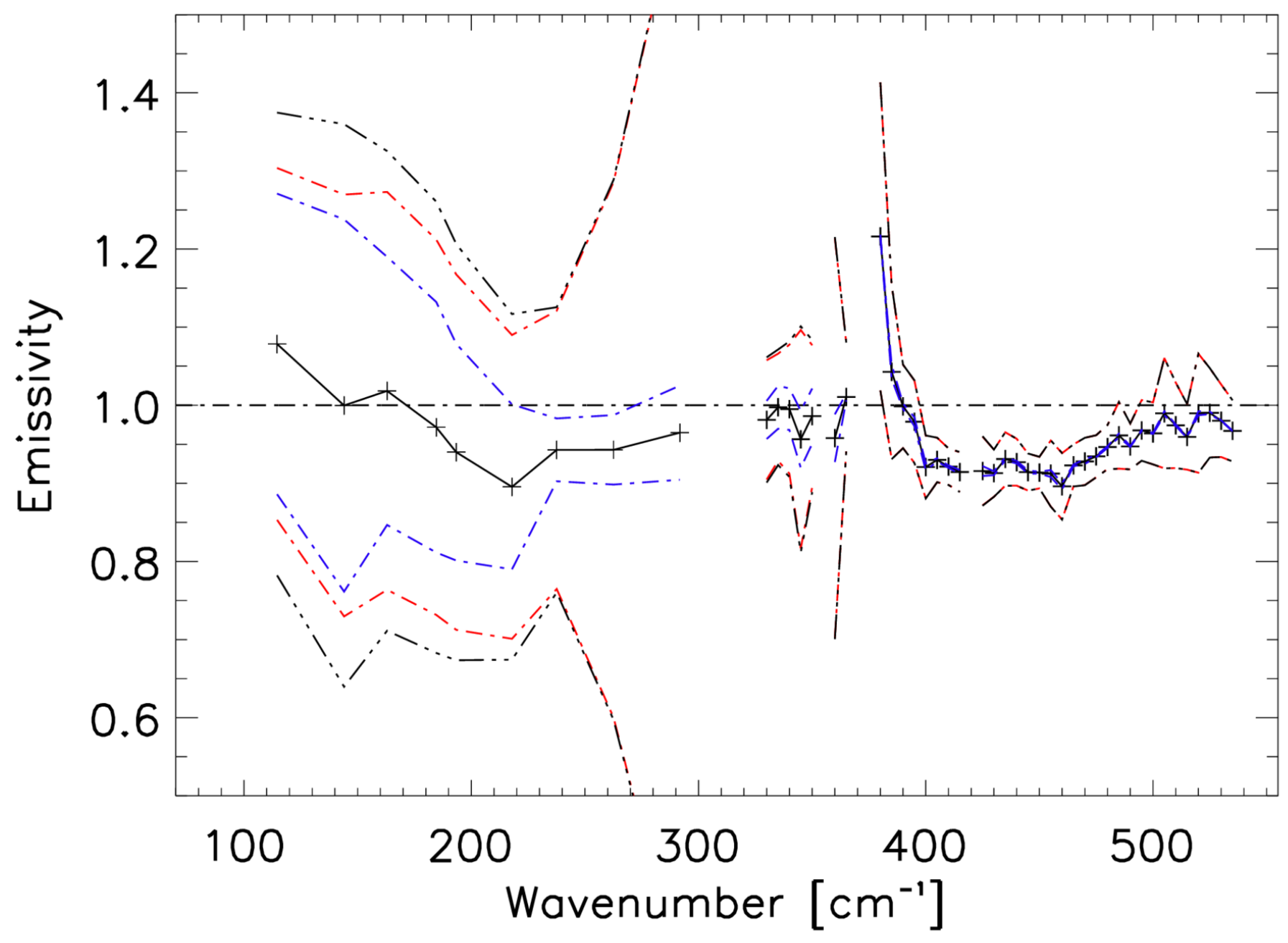

2017JD027328-f11-z-.tif 

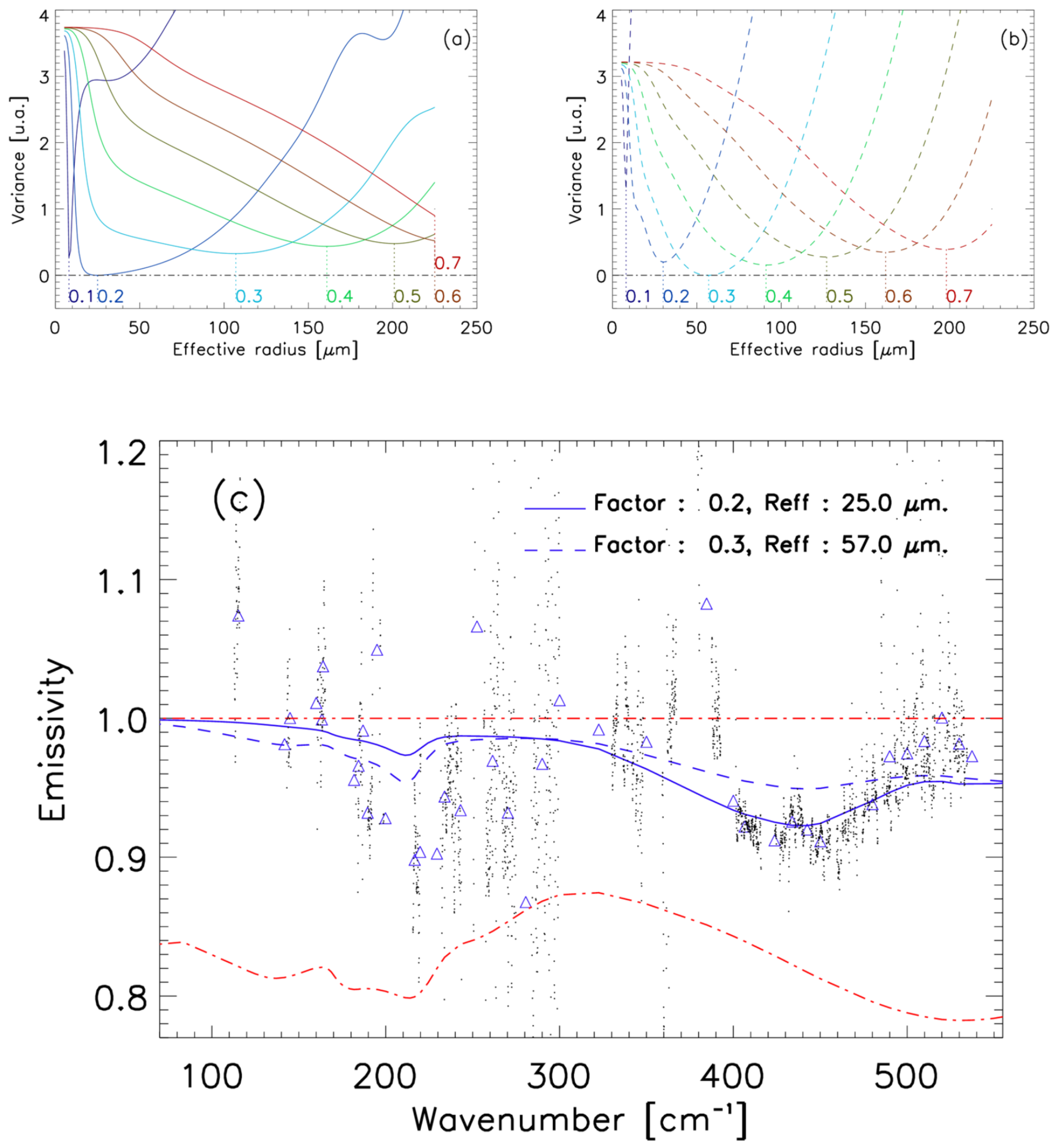

2017JD027328-f12-z-.tif 

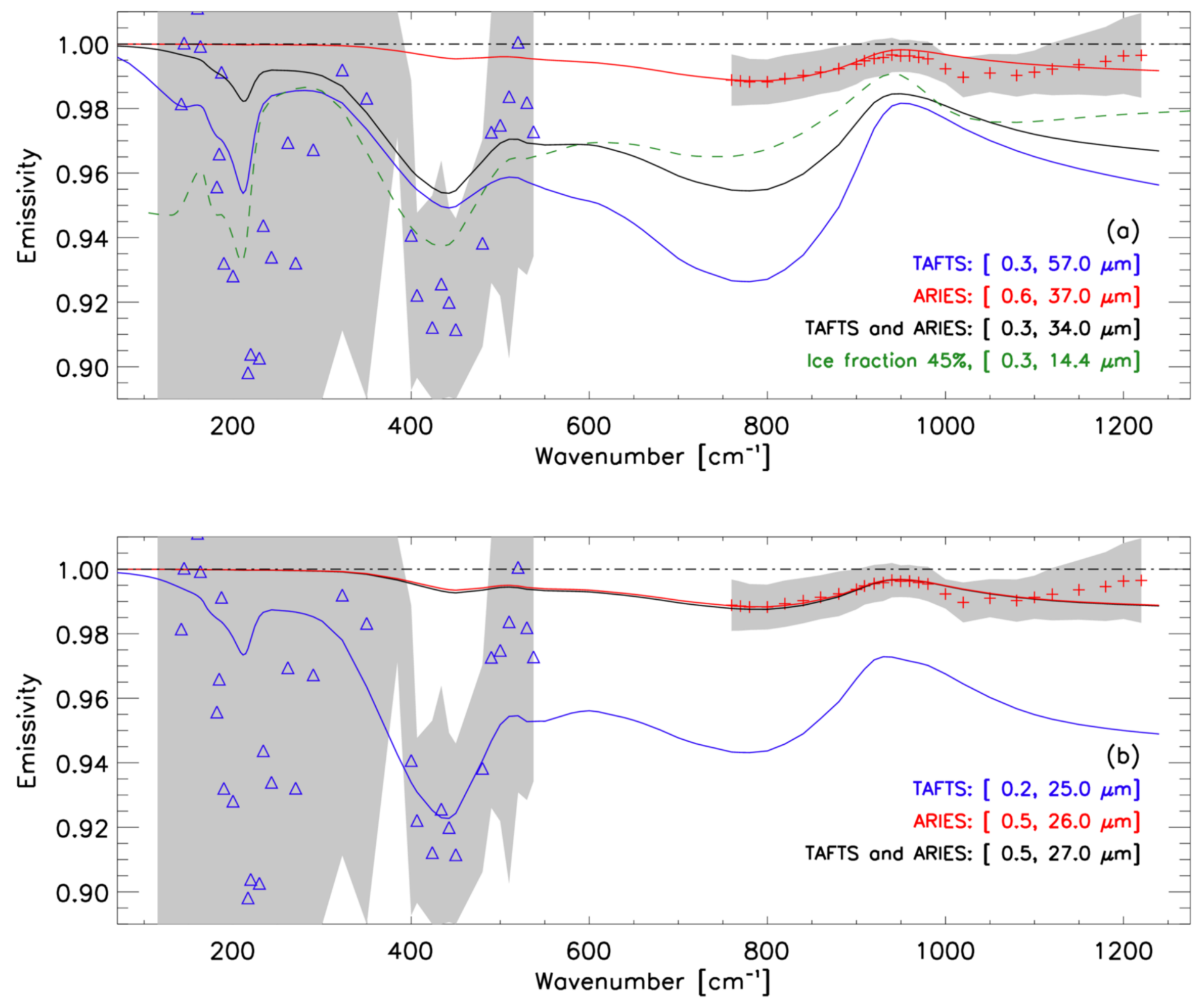

2017JD027328-f13-z-.tif 